\title{
Turbulent Wake Field Reconstruction of VLCC Models Using Two-dimensional Towed Underwater PIV Measurements
}

by

Jeonghwa Seo ${ }^{1}$, Dong Myung Seol ${ }^{2}$, Bumwoo Han ${ }^{3}$ and Shin Hyung Rhee ${ }^{1,4+}$

${ }^{1}$ Dept. of Naval Architecture and Ocean Engineering, Seoul National University, Seoul, Korea

${ }^{2}$ Defense Acquisition Program Administration, Seoul, Korea

${ }^{3}$ Hyundai Heavy Industries, Co., Ltd., Seoul, Korea

${ }^{4}$ Research Institute of Marine Systems Engineering, Seoul National University, Seoul, Korea 
${ }^{+}$Corresponding Author, Tel: +82-2-880-1500, Fax: +82-2-888-9298, Email: $\underline{\text { shr@ snu.ac.kr }}$

\begin{abstract}
In the present study, the wake fields of two full ship models were measured using a twodimensional (2D) towed underwater particle image velocimetry (PIV) system to identify the flow characteristics around a hull with a high block coefficient. The 2D PIV measurements and reconstructing the three-dimensional (3D) flow field by stacking the $2 \mathrm{D}$ measurements were validated and applied to the model ship wakes in a towing tank. The bare hull wake was measured for a very large crude oil carrier (VLCC) model, KVLCC2, at a Froude number of 0.142 based on the length between perpendiculars $\left(L_{P P}\right)$. These measurements were compared with those of five-hole Pitot tubes, a Pitot-static tube, and hot-wire anemometers for validation. After the validation tests, the nominal wake field of a generic VLCC model with a Froude number of 0.145 based on $L_{P P}$ was measured. To identify the effects of a rudder and rotating propeller on the mean velocity and turbulence characteristics, a 3D reconstruction was carried out for the generic VLCC model under self-propulsion conditions.
\end{abstract}

Keywords: Particle image velocimetry, Towing tank experiment, Ship wake measurement 


\section{INTRODUCTION}

Improving the propulsion and maneuvering performance of a ship requires a quantitative understanding of flow around the ship, as well as a well-designed propeller and rudder. The stern flow field is very complex owing to 3D flow separation, the development of a turbulent boundary layer, and the orbital motion of water particles induced by free surface waves. To investigate the wake field of ships, various experimental studies have been attempted and reliable computational methods have been developed and applied.

In experimental naval hydrodynamics, a five-hole Pitot tube is the most commonly used device for measuring velocity fields, especially in towing tank facilities. Using five-hole Pitot tubes, numerous wake measurements have been conducted for various types of ships, and some of the model test results have been provided as validation data for computational fluid dynamics (CFD): Series 60 cargo ship (Toda et al., 1990; Toda et al., 1992; Longo and Stern, 2002), a container ship and a VLCC designed by Korea Research Institute of Ships and Ocean engineering (KRISO) (Kim et al., 2001; Yang et al., 2009), a liquefied natural gas carrier (Van et al., 2006), and a surface combatant (Longo and Stern, 2005).

Pitot tube systems only yield point-wise measurements of the velocity field with timeaveraged pressure differences; therefore, they cannot measure the parameters that are significant for turbulence studies, e.g., the Reynolds stresses or turbulent kinetic energy. Given that propellers and rudders work in fully developed turbulent wake fields, it is essential to investigate turbulent wake characteristics using model tests; thus, a great demand exists for novel experimental techniques that can measure the fluctuating components of velocity and turbulence characteristics.

Hot-wire anemometry is one of the alternatives to the Pitot tube system. Although it is also a point-wise measurement system with limitations in the measurable flow angle, it precisely 
measures fluctuations in turbulent flows with high repetition rate. The flow fields around a double-body model of the KRISO VLCC (KVLCC) and container ship (KCS) were measured in a wind tunnel with hot-wire anemometers (Lee et al., 2003a). The corresponding Reynolds numbers for the wind tunnel tests were identical to those in towing tank experiments with the same test model (Kim et al., 2001); thus, the characteristics of different measurement systems could be compared. Moreover, the time-averaged velocity and turbulence properties obtained from the wind tunnel test were used as validation data for CFD (e.g., Park et al., 2015).

Laser Doppler velocimetry (LDV) systems have been employed to measure the flow fields around ships and propellers in towing tanks and cavitation tunnels. The flexibility in arrangements enabled the measurement of complex flow behavior such as the upstream flow of a rotating propeller behind a ship model (Felli and Di Felice, 2005) and propeller-rudder interactions (Felli et al., 2009). Tests were able to measure significant and precise flow characteristics in turbulent wake fields; however, as both the hot-wire and LDV methods use point-wise velocity measurements, they are quite time-consuming and challenging to apply to routine towing tanks tests.

PIV is one of the newest experimental techniques in naval hydrodynamics. 2D PIV measurements were performed for model tests in circulating water channels: a KCS model in bare hull conditions (Lee et al., 2003b), with a rotating propeller (Paik et al., 2004), and various loading conditions (Lee et al., 2009). Besides 2D PIV measurements, stereoscopic PIV (SPIV) measurements were conducted in circulating water channels for the wake field of a Series 60 model with a rotating propeller (Calcagno et al., 2005) and propeller wake in open water (Lee et al., 2004). The flow characteristics of propellers have been measured in cavitation tunnels: a propeller wake field (Cotroni et al., 2000), the velocity fields around a rotating propeller with various advance ratios (Paik et al, 2007), a propeller in a simulated hull wake (Paik et al., 2010), and a propeller with a rudder (Felli and Falchi, 2011). 
Although PIV systems in cavitation tunnels and circulating water channels have yielded extensive results for naval hydrodynamics, they are limited by blockage owing to the constrained cross-sectional area of the test and initial turbulence in the free stream. To overcome these limitations, PIV systems have been introduced to towing tanks. These PIV systems require specially designed optical components and tracer particle seeding techniques for underwater operations. Despite these difficulties, several facilities have adopted towing tank PIV systems. The flows have been measured around model ships, such as the DTMB 5512 model: in calm water (Gui et al., 2001), in regular head waves (Longo et al., 2007), and under planar motion mechanism (PMM) maneuvers (Yoon, 2009). Furthermore, PIV and SPIV systems in towing tanks have measured the trajectory of a wake vortex generated from a model airplane (Scarano et al., 2002), a phase-averaged propeller wake (Anschau and Mach, 2007), and the flow around a twin-skeg ship and an energy saving device (Nagaya et al., 2011). PIV systems in towing tanks experience an environment that is different from cavitation tunnels and water channels; consequently, Grizzi et al. (2010) have suggested specialized calibration techniques based on uniform flow measurements.

In the present study, the wake fields of two full ship models were measured using a towed underwater 2D PIV system to identify the flow characteristics with a high block coefficient $C_{B}$ (i.e., the ratio of the displacement of a ship to the surrounding cuboid). To validate the measurement system, a nominal wake field for the KVLCC2 model was measured using the PIV system and compared with the results of Pitot tube and hot-wire anemometry measurements, which are currently provided as validation data for CFD by the International Towing Tank Conference (ITTC). To obtain the nominal wake field that is perpendicular to the longitudinal direction, 2D vectors on the horizontal and vertical measurement planes (from 2D PIV measurements) were stacked to reconstruct a 3D volume and extract the nominal wake field. Next, the nominal wake field of a generic tanker model with a similar $C_{B}$ 
was measured and compared to the results of the KVLCC2. Furthermore, a stern flow field under self-propulsion conditions was measured to investigate propeller-hull interactions.

This study is organized as follows. In Section 2, the test facility, equipment, and analysis procedures for the PIV system are described. Then, in Section 3, the test models, conditions, and specific locations of fields-of-view are depicted, followed by validation with the KVLCC2 model. Test results for a generic tanker model with and without a propeller and rudder are presented and discussed in Section 4. Finally, the results are summarized and conclusions are presented in Section 5. 


\section{TESTING FACILITY, EQUIPMENT, AND DATA ACQUISITION}

The 2D PIV measurements were conducted in the Seoul National University towing tank (SNUTT), which is 110-m long, 8-m wide, and 3.5-m deep. The test model and 2D PIV system were installed on a towing carriage with a maximum speed of $5 \mathrm{~m} / \mathrm{s}$ and a speed control tolerance less than $0.002 \mathrm{~m} / \mathrm{s}$.

The underwater 2D PIV system at SNUTT is shown in Fig. 1; it was designed and manufactured by Dantec Dynamics (Skovlunde, Denmark). The system comprised a 50-mJ, dual-headed Nd:Yag laser (pulse rate: $15 \mathrm{~Hz}$ per laser head) and a Dantec Flowsense 2.0M $1600 \times 1200$-pixel digital camera fitted with a NIKKOR f/1.4 50-mm lens. The camera module and cylindrical lenses created a 2-mm-thick laser sheet; they were placed in separate waterproof cases, which had a streamlined shape to minimize disturbances to the nearby flow field. Positioning of the whole system was handled by an automated traversing system manufactured by Dantec Dynamics and errors in the positioning was less than $0.01 \mathrm{~mm}$. It moved the PIV housing along the traverse and vertical rails. At the origin of the coordinate system, a pin was put as a marker for PIV measurement.

The distance from the camera lens to the field-of-view was fixed at $550 \mathrm{~mm}$. The size of the field-of-view was $102.08 \times 76.56 \mathrm{~mm}$ and within the field of view, and one pixel physically occupied an area of $63.8 \times 63.8 \mu \mathrm{m}$. Polyamide particles with a density of 1030 $\mathrm{kg} / \mathrm{m}^{3}$ and an average diameter of $27 \mu \mathrm{m}$ were used as tracer particles. A mixture of $1 \mathrm{~L}$ of particles and $40 \mathrm{~L}$ of water was seeded in the towing tank prior to testing.

In the present study, a total of three test conditions were selected: a nominal wake measurement for each of the two model ships and a wake analysis for a model under selfpropulsion conditions. For each test condition, the 2D flow fields were measured using PIV for 36-40 carriage runs; this could be achieved in a single day, with 15 mins of wait time 
between carriage runs. The temperature change of the water was negligibly small, less than $0.5^{\circ} \mathrm{C}$ in a day of tests.

The software Dynamic studio V2.2 from Dantec Dynamics was used to capture the particle images and analyze the velocity fields. For the self-propulsion test, the trigger of the PIV measurement system was synchronized with propeller rotation to acquire phase-averaged velocity fields. The rotational displacement of the propeller was measured using a rotary encoder with a 12-bit resolution and an uncertainty of $0.088^{\circ}$. During the capture of a two particle image frames, the propeller rotated $1.44^{\circ}$. For the self-propulsion test, the propeller phase was varied from $0^{\circ}$ to $75^{\circ}$ at intervals of $15^{\circ}$, enabling the characterization of the wake field for six different propeller phases.

The size of the interrogation window was $32 \times 32$ pixels. The interrogation windows overlapped by $50 \%$ so that one velocity vector represented the mean velocity of an area of $1.03 \times 1.03 \mathrm{~mm}$ on the measurement plane. Velocity vectors were computed by cross correlation with the multi-pass method, and a moving average validation filter was applied to remove spurious vectors. For a single towing carriage run, 250 particle image pairs were acquired and Reynolds averaged to derive two in-plane components of the average velocity, two normal components of the Reynolds stresses, and one shear component. An uncertainty assessment of the PIV system, based on the uniform flow results (Seol et al., 2013), is reported in Table 1.

A 3D flow field reconstruction method for a towed, underwater, 2D PIV system was initially suggested by Gui et al. (2001). The volumetric reconstruction procedure is shown in Fig. 2. To reconstruct a 3D vector field, 2D flow fields on the horizontal and vertical planes were measured in increments of $4.9 \mathrm{~mm}$; then, the 2D flow measurements were stacked. A set of results for the horizontal planes was linearly interpolated to form a 3D flow field, and the same interpolation procedure was applied to the vertical plane results. Vectors on vertical and 
horizontal planes were combined to a single, three-component $(3 \mathrm{C})$ velocity vector.

$$
\begin{aligned}
& \overline{\boldsymbol{u}_{v}}=\left(\overline{u_{v}}, 0, \overline{w_{v}}\right), \\
& \overline{\boldsymbol{u}_{h}}=\left(\overline{u_{h}}, \overline{v_{h}}, 0\right), \\
& \overline{\boldsymbol{u}}=\left(\frac{\overline{u_{v}}+\overline{u_{h}}}{2}, \overline{v_{h}}, \overline{w_{v}}\right),
\end{aligned}
$$

where $U, \overline{\boldsymbol{u}_{v}}$, and $\overline{\boldsymbol{u}_{h}}$ are the towing speed of the test model and the Reynolds-averaged velocity vectors for the vertical and horizontal planes, respectively. It should be noted that the standard deviation of the disparity between $\overline{u_{v}}$ and $\overline{u_{w}}$ at most locations was less than $2 \%$ $U$. The disparity due to the positioning of the measurement planes was $2.5 \% U$ at most. Disparities between the horizontal and vertical plane measurements of the axial velocity and turbulence properties are described in the results section.

Each component of the Reynolds normal stress was derived from the difference between the instantaneous speed $u_{i}$ and Reynolds-averaged velocity from one carriage run $\bar{u}$ as follows. The axial components of the Reynolds normal stress in the vertical and horizontal planes were averaged during the flow field reconstruction.

$$
\begin{aligned}
& \overline{u^{\prime} u^{\prime}}=\frac{1}{250} \sum_{i=1}^{250}\left(u_{i}-\bar{u}\right)\left(u_{i}-\bar{u}\right) \\
& \overline{v^{\prime} v^{\prime}}=\frac{1}{250} \sum_{i=1}^{250}\left(v_{i}-\bar{v}\right)\left(v_{i}-\bar{v}\right) \\
& \overline{w^{\prime} w^{\prime}}=\frac{1}{250} \sum_{i=1}^{250}\left(w_{i}-\bar{w}\right)\left(w_{i}-\bar{w}\right)
\end{aligned}
$$

The turbulence kinetic energy, $k$, was also calculated from the PIV results. 


$$
k=\frac{1}{2}\left(\overline{u^{\prime} u^{\prime}}+\overline{v^{\prime} v^{\prime}}+\overline{w^{\prime} w^{\prime}}\right)
$$

In addition, the Reynolds shear stress was derived. It is the cross correlation between the two fluctuating velocity components; thus, only the $x-y$ and $x-z$ components were identified in the horizontal and vertical 2D vector fields, respectively.

$$
\begin{aligned}
& \overline{u^{\prime} v^{\prime}}=\frac{1}{250} \sum_{i=1}^{250}\left(u_{i}-\bar{u}\right)\left(v_{i}-\bar{v}\right) \\
& \overline{u^{\prime} w^{\prime}}=\frac{1}{250} \sum_{i=1}^{250}\left(u_{i}-\bar{u}\right)\left(w_{i}-\bar{w}\right)
\end{aligned}
$$

For comparison with the 2D PIV results, a Pitot-static probe was used to measure the axial velocity in the nominal wake. The outer diameter of the probe was $2 \mathrm{~mm}$, and the diameter of the orifice at the front end stagnation point was $1 \mathrm{~mm}$. A DP-15 pressure transducer (Validyne engineering, Northridge, CA), CD-18 amplifier from Validyne engineering, and an NI6225 data acquisition system from National Instruments (Austin, TX) were used to measure the pressure differences between orifices and thereby derive the axial velocity using the Bernoulli equation:

$$
\bar{u}=\sqrt{2 g \Delta h}
$$

where $g$ is the gravitational acceleration and $\Delta h$ is the hydraulic head measured by the pressure transducer. 


\section{TEST MODELS AND CONDITIONS}

In this study, a KVLCC2 model, which is a modification of the KVLCC model to improve the flow around its stern (Kim et al., 2001) was used to validate the 3D3C velocity field reconstruction technique. The model length was $1 / 100$ of the prototype, and its surface was painted in matte black to reduce the reflection of the laser sheet during testing. An array of studs was attached at the bulbous bow and station 19 , following the standard recommendation of ITTC to stimulate turbulent flow. Note that stations 0 and 20 were located in the aft and fore perpendiculars, respectively. The body plan and profile of the KVLCC2 are shown in Fig. 3 , and its principal dimensions are listed in Table 2. The blockage ratio from the crosssectional area of the model and that of the towing tank was $0.43 \%$, which is negligibly small. Therefore, no speed correction for the blockage effect was applied. The towing speed was $0.797 \mathrm{~m} / \mathrm{s}$, i.e., equivalent to 15.5 knots for a full-scale ship. For the model scale conditions, the corresponding Reynolds and Froude numbers based on the length between perpendiculars $\left(L_{P P}\right)$ were $2.32 \times 10^{6}$ and 0.142 , respectively. The vertical position of the KVLCC2 model was fixed at an even keel, with no trim or sinkage, for comparison with other experimental results. The stern flow of the bare hull was measured by 2D PIV and a Pitot-static tube; no dummy hub cap was attached in the location of the propeller.

A right-handed Cartesian coordinate system was used with the origin located at the propeller center on station 0.35 . The positive $x, y$, and $z$ directions were downstream, starboard, and upward, respectively. Lengths were non-dimensionalized by the radius $(R)$ of the propeller. Horizontal measurement planes were located from $z / R=-1.1$ to 1.1 with a spacing of $0.1 R(4.9 \mathrm{~mm})$, whereas vertical planes were located from $y / R=-0.1$ to 1.1 with the same spacing as that for the horizontal planes. The locations of the planes were carefully chosen to include the propeller disk area for comparison with existing experimental data. For 


\section{VALIDATION WITH KVLCC2}

The results at the propeller plane, i.e., station 0.35, were obtained and compared. All measured and derived variables, including the length, velocity, vorticity, and Reynolds stresses, were non-dimensionalized by $R, U$, the propeller radius divided by towing speed $R / U$, and the square of the towing speed $U^{2}$.

The disparity in measurements between the horizontal and vertical planes was evaluated first. The values of $\overline{u_{h}} / U$ and $\overline{u_{v}} / U$ from the nominal wake measurements of the KVLCC2 model ship are compared in Figs. 4 and 5. The standard deviation of the disparity between $\overline{u_{h}} / U$ and $\overline{u_{v}} / U$ in the nominal wake plane was approximately $1.54 \% U$. Disparities in the axial velocity were mainly observed where the gradient of $\bar{u}$ was large. This implies that errors in locating the measurement planes could affect the uncertainty in $3 \mathrm{D}$ reconstruction.

The contours of the axial velocity and velocity vectors in the $y-z$ plane at station 0.35 are shown in Fig. 6. Hook-shaped axial velocity contours were clearly observed in the region of the bilge vortex; this is a well-known characteristic of the wake field of a full ship. In addition, a pair of counter-rotating vortices existed just under the propeller boss, where the axial velocity was less than $10 \%$ of the towing speed. The iso-surfaces of axial velocity in the measurement volume are shown in Fig. 7 for $u / U=0.3,0.5$, and 0.7. The iso-surface for $u / U$ $=0.3$ contracts in the downstream, implying that the axial velocity recovers in the wake.

The results of the 3D flow field reconstruction are compared with measurements from Pitot tubes and hot-wire anemometers in Fig. 8. The hot-wire test excluded free surface wave effects by using a double-body model; nevertheless, the four set of results showed good agreement. Ships designed for operation in low-Froude number conditions, e.g., VLCCs, generate relatively short and small waves around the hull. These wave effects are generally confined to the region near the free surface and hardly affect the flow field near the propeller. 
The streamlines and contours of the non-dimensionalized axial vorticity $\left(\zeta_{x} R / U\right)$ in the propeller plane are shown in Fig. 9. The streamlines clearly indicate rotating flow at the vortex centers. Two significant rotational flows existed in the wake plane: one in the region of the bilge vortex and the other below the propeller boss. The vortex below the propeller boss was confined to a small region, and the magnitude of its vorticity was greater than 30 , which is twice that of the bilge vortex.

To assess the reliability of turbulence property measurements made with the PIV system, the vertical and horizontal plane measurements of $\overline{u^{\prime} u^{\prime}} / U^{2}$ were compared, as shown in Figs. 10 and 11. In general, the disparity in the measurements of $\overline{u^{\prime} u^{\prime}} / U^{2}$ was larger than that of $\bar{u} / U$, as the uncertainty in the measurement of turbulence properties was larger than that of mean velocity. The standard deviation of the disparity was $0.22 \% U^{2}$.

Both $k / U^{2}$ and each component of the Reynolds stresses in the nominal wake field are shown in Fig. 12. The Reynolds normal stress and $k / U^{2}$ were concentrated in the region, where the gradient of the axial velocity was large and magnitude of its local maxima was greater than 0.028. It should be noted that turbulence around the stern was anisotropic. The axial velocity dominated and $\overline{u^{\prime} u^{\prime}} / U^{2}$ had the largest magnitude among the three components. Moreover, note that $\overline{u^{\prime} v^{\prime}} / U^{2}$ and $\overline{u^{\prime} w^{\prime}} / U^{2}$ were much weaker than $\overline{v^{\prime} v^{\prime}} / U^{2}$ and $\overline{w^{\prime} w^{\prime}} / U^{2}$.

The values of $k / U^{2}$ from PIV and hot-wire measurements are compared in Fig. 13. The PIV results were quite similar to the hot-wire results, except that the local maximum of $k / U^{2}$ at $0.8 R$ was 0.028 for the PIV results, which is twice the value of the hot wire measurement. Differences in the measurement characteristics may account for the disparity, as the PIV system had a lower repetition rate for data acquisition and a smaller sample size than the hotwire measurement. 


\section{WAKE MEASUREMENT RESULTS: A GENERIC TANKER}

The wake field measurement in the propeller plane (in the absence of the propeller) is shown in Fig. 14 for the generic tanker. The local maximum in axial velocity had a value of $0.33 U$ (i.e., larger than that of the KVLCC2, $0.26 U$ ) in the region of the hook-shaped wake contours. In addition, the velocity distribution was more uniform with smaller gradients than those in the KVLCC2. Bilge and hub vortices were also observed at locations similar to those in the KVLCC2 profile.

The distributions of $k / U^{2}$ and $\overline{u^{\prime} u^{\prime}} / U^{2}$ in the propeller plane are shown in Fig. 15; they are similar to those of the KVLCC2 model. $k / U^{2}$ was concentrated where the gradient of the axial velocity was large, and the non-dimensionalized magnitude of the local maximum was 0.022 (slightly lower than that of the KVLCC2, 0.028). It appears that the gradient of the axial velocity is closely related to turbulence characteristics; therefore, minimizing this gradient is beneficial for both making the wake field uniform and reducing the magnitude of $k / U^{2}$ at the propeller plane.

The propeller wake was measured in the self-propulsion condition (with the rudder and propeller boss cap present), as shown in Fig. 16. Prior to reconstructing 3D flow fields, representative 2D flow fields were analyzed to determine the characteristics of the wake field behind a working propeller. First, 2D phase-averaged and time-averaged flow fields were analyzed at $z / R=0$. The phase-averaged $2 \mathrm{D}$ velocity field of a horizontal plane at $z / R=0$ is shown in Fig. 17. For phase-averaging, 20 instantaneous velocity fields were selected at the same propeller phase.

The velocity fields were analyzed by Galilean decomposition with the axial transport velocity of $1.0 U$. The origin was at the center of the rotating propeller, and the region where the laser sheet was blocked by the rudder was masked. The periodic appearance of tip vortices along the boundary of the propeller wake and development of the wake field were 
observed in the Galilean decompositions. The locations of the tip vortices could be identified; the transfer speeds of tip vortices, derived from the locations of second and third tip vortices, were found to be $1.01 \mathrm{U}$ on the port side and $0.97 \mathrm{U}$ on the starboard side. The different transfer speeds indicate that the downstream propeller wake was stretched on the port side.

The $u / U$ distribution of the horizontal plane is shown in Fig. 18. At the location of the tip vortices, $u / U$ changed rapidly. Interestingly, the local maxima of axial velocity on the port and starboard sides were different; the magnitude of the local maximum was $1.18 U$ and $1.33 U$ on the port and starboard sides, respectively. This is attributed to differences in the angle of attack onto the propeller blades and magnitude of the inflow velocity on the starboard and port sides, as shown in Fig. 19. For the propeller selected, the angle of attack and magnitude of the inflow velocity at $r / R=0.7$ were $3.92^{\circ}$ and $3.05 U$, respectively, for open water conditions. When the same propeller was rotating in the ship hull wake, however, the presence of the hull and propeller-induced flow in front of the propeller altered the angle of attack and inflow velocity magnitude. The mean inflow velocity in the wake $(2.45 U)$ was slightly less than that in open water $(2.55 \mathrm{U})$, obviously owing to the presence of the hull; however, the decrease in the inflow velocity magnitude was relatively small on the starboard side and contributed to the higher-valued local maximum in $u / U$ on the starboard side.

The time-averaged axial velocity distribution in the horizontal plane $(z / R=0)$ is shown in Fig. 20. Similar to the phase-averaged results in Fig. 18, the flow field on the starboard side had a higher loading on the propeller blade and a larger gradient in the axial velocity near the boundary of the propeller wake. The time-averaged $u / U$ profiles in the horizontal plane $(z / R=$ 0) are shown in Fig. 21 at stations 0.23, 0.16, and 0, which correspond to the rudder bulb, leading edge of the rudder, and rudder stock, respectively. The propeller action, shown by the increase in $u / U$, was mostly concentrated in the region within $r / R=0.8$. The velocity increase was clearly greater on the starboard side, which resulted from the greater propeller loading. 
The non-dimensionalized vorticity distribution and locations of tip vortices in the horizontal plane are shown in Fig. 22. Furthermore, the magnitude of the vorticity was greater on the starboard side because of the stronger rounding-up flow around the blade tip, which confirmed that the propeller loading was greater on the starboard side.

The flow structure in the vertical planes is displayed in Fig. 23 by the magnitude of vorticity at different transverse locations. Tip vortices in the upper half were more irregular than those in the lower half and were transported downstream asymmetrically. The reason for the irregularity and asymmetry was the stronger 3D flow in the upper half, induced by the low inflow velocity on the propeller blades and bilge vortices on top of the propeller hub vortices. Owing to the irregular and asymmetric flow structure in the vertical planes, more severe noise and vibration problems were generated in the upper half of the wake, even though the propeller loading was lighter on the upper half. It is worth mentioning that the wake contracted more on the starboard side owing to the higher velocity in the propeller wake.

The hub vortex was observed near the propeller hub (Fig. 23), i.e., $y / R=-0.2$ and 0.2 ; however, it weakened quickly $(y / R=-0.3$ and 0.3$)$ and eventually disappeared $(y / R=-0.4$ and 0.4) with increasing distance from the hub. Notably, the stronger hub vortex was on the port side, where the propeller loading was relatively low and propeller rotation direction enhanced vortex generation in the clockwise direction.

The iso-surfaces of helicity in the reconstructed 3D flow field are shown in Fig. 24. The tip vortex trajectory identified by high helicity is shown only on the port side, because it was hard to identify on the starboard side owing to severe distortion in the wake.

The reconstructed 3D flow field is displayed in Figs. 25 and 26. The time-averaged velocity components and $k / U^{2}$ are shown for cross sections at stations $0.23,0.16$, and 0 , which correspond to the rudder bulb, leading edge of the rudder, and rudder stock, 
respectively. Contours of $u / U$ clearly showed that the speed increased and propeller wake area shrunk along the downstream direction. Note that the speed increase was greater on the starboard side. The cross-flow $(v / U-w / U)$ vectors were more interesting. Hook-shaped wake contours were still observed on the starboard side. The port side bilge vortex merged with the propeller's rotational flow, which was in the same clockwise direction. Although less prominent than in the nominal wake, the bilge vortex survived on the starboard side and caused an undesirable cross-flow pattern in the upper-right region of the propeller wake. The presence of the rudder caused the flows on the port and starboard sides to separate, thereby limiting the propeller action on each side.

The contours of $k / U^{2}$ in Fig. 26 were similar at all three locations, i.e., they had a large magnitude where the velocity gradients were large, which was observed almost entirely on the starboard side. The upper starboard side had an especially high value of $k / U^{2}$, which resulted from violent fluctuations owing to rapid changes in the transverse and vertical velocity components of the propeller wake. 


\section{CONCLUSIONS}

To investigate the turbulent wake fields of full ships, the wake fields of a KVLCC2 model and a generic tanker model were measured using a 2D PIV system in a towing tank. These two hulls of similar geometry had similar operating conditions, and therefore, this study focused on understanding the hydrodynamic characteristics of the wake fields of full ships with large block coefficients.

The PIV system and reconstruction technique were validated by comparing measurements of the mean velocity and turbulence properties for the KVLCC 2 model to other experimental results. The two sets of results showed good agreement in terms of the axial velocity and development of vortices. A 3D3C flow field reconstruction was applied to compare the nominal wake fields.

The wakes of a generic tanker model in the bare hull and self-propulsion conditions were measured using the experimentally validated system and methods. In the bare hull condition, the measured data were similar to those obtained with the KVLCC2 model. In the selfpropulsion condition, the reconstruction method demonstrated the feasibility of capturing the influence of the propeller and rudder. The propeller loading was found to be unbalanced because of the propeller rotation in the irregular hull wake, propeller tip vortices, flowdividing effect of the rudder, and asymmetric and distorted structure of the propeller wake.

Based on these results, the reconstruction method proved to be an inexpensive and reasonable way of measuring the full ship wakes, even in the presence of a rotating propeller and its appendages. However, care should be taken in employing the reconstruction method, especially when 3D effects are strong. 


\section{ACKNOWLEDGEMENTS}

This research was supported by the IT R\&D program of MOTIE/KEIT (Grant No. 100660329), the National Research Foundation of Korea, grant funded by the Korean government (Grant Nos. 2013R1A1A2012597, 2011-0020563, and 2009-0083510), and U.S. Office of Naval Research (ONR) and ONR Global as part of the Naval International Cooperative Opportunities in Science and Technology Program (NICOP) under the supervisions of Drs. Thomas Fu and Woei-Min Lin. The assistance on model tests from Geuk-sang Yoo and Changhee Kim of Seoul National University is gratefully acknowledged. 


\section{REFERENCES}

Anschau, P. and Mach, K. P., 2007. “Application of a stereo PIV system for investigation of flow fields in towing tank and cavitation tunnel," Archives of Civil and Mechanical Engineering, Vol. 7, No. 3, pp. 5-17.

Calcagno, G., Di Felice, F., Felli, M., Pereira, F., 2005. “A stereo-PIV investigation of a propeller's wake behind a ship model in a large free-surface tunnel," Marine Technology Society Journal, Vol. 39, No. 2, pp. 94-102.

Controni, A., Di Felice, F., Romano, G. P., Elefante, M., 2000. "Investigation of the near wake of a propeller using particle image velocimetry," Experiments in Fluids, Vol. 29, No. 1, pp. 227-236.

Felli, M. and Di Felice, F., 2005. "Propeller wake analysis in nonuniform inflow by LDV phase sampling techniques," Journal of Marine Science and Technology, Vol. 10, No. 4, pp. 159-172.

Felli, M., Roberto, C., Guj, G., 2009. "Experimental analysis of the flow field around a propeller-rudder configuration,” Experiments in Fluids, Vol. 46, No. 1, pp. 147-164.

Felli, M. and Falchi, M., 2011. "Propeller tip and hub vortex dynamics in the interaction with a rudder," Experiments in Fluids, Vol. 51, No. 5, pp. 1385-1402.

Grizzi, S., Pereira, F., Di Felice, F., 2010, “A simplified, flow-based calibration method for stereoscopic PIV," Experiments in Fluids, Vol. 48, No. 3, pp. 473-486.

Gui, L., Longo, J., Stern, F., 2001. “Towing tank PIV measurement system, data, and uncertainty assessment for DTMB Model 5512," Experiments in Fluids, Vol. 31, No. 3, pp. 336-346.

Kim, W., Van, S., Kim, D., 2001. "Measurement of flows around modern commercial ship models," Experiments in Fluids, Vol. 31, No. 5, pp. 567-578. 
Lee S., Kim, H., Kim, W., Van, S., 2003a. "Wind tunnel tests on flow characteristics of KRISO 3600TEU container ship and 300K VLCC ship double models," Journal of Ship Research, Vol. 47, No. 1, pp. 24-38.

Lee, S., Koh, M., Lee, C., 2003b. "PIV velocity field measurements of flow around a KRISO 3600TEU container ship model," Journal of Marine Science and Technology, Vol. 8, No. 2, pp. 76-87.

Lee, J., Paik, B., Lee, S., 2009. "PIV measurements of hull wake behind a container ship model with varying loading condition," Ocean Engineering, Vol. 36, No. 5, pp. 377-385.

Lee., S., Paik, B., Yoon, J., Lee, C., 2004. “Three-component velocity field measurements of propeller wake using a stereoscopic PIV technique," Experiments in Fluids, Vol. 36, No. 4, pp. 575-585.

Longo, J. and Stern, F., 2002. "Effects of drift angle on model ship flow," Experiments in Fluids, Vol. 32, No. 5, pp. 558-569.

Longo. J. and Stern, F., 2005. "Uncertainty assessment for towing tank tests with example for surface combatant DTMB model 5415," Journal of ship research, Vol. 49, No. 1, pp. 5568.

Longo, J., Shao, J., Irvine, M., Stern, F., 2007. "Phase-averaged PIV for the nominal wake of a surface ship in regular head waves," ASME Journal of fluids engineering, Vol. 129, No. 5, pp. 524-540.

Nagaya, S., Ochi, F., Fukui, Y., Omori, T., Inukai, Y., 2011. “Stereo PIV measurements of flow around energy saving device and twin-skeg stern ship in towing tank," Proceedings of Advanced model measurement technology for EU maritime industry, Newcastle upon Tyne, UK, April, 2011.

Paik, B., Lee, C., Lee, S., 2004. "PIV analysis of flow around a container ship model with a rotating propeller," Experiments in Fluids, Vol. 36, No. 6, pp. 833-846. 
Paik, B., Kim, J., Park, Y., Kim, K., 2007. “Investigation on the vortex structure of propeller wake influenced by loading on the blade," Journal of Marine Science and Technology, Vol. 12, No. 2, pp. 72-82.

Paik, B., Kim, K., Lee, J., Lee, S., 2010. "Analysis of unstable vertical structure in a propeller wake affected by a simulated hull wake," Experiments in Fluids, Vol. 48, No. 6, pp. $1121-1133$.

Park, S., Oh, G., Rhee, S. H., Koo, B. -Y., and Lee, H., "Full scale wake prediction of an energy saving device by using computational fluid dynamics,” Ocean Engineering, Vol. 101, pp. 254-263.

Scarano, F., van Wijk, C., Veldhuis, L.L.M., 2002. "Traversing field of view and AR-PIV for mid-field wake vortex investigation in a towing tank," Experiments in Fluids, Vol.33, No.6, pp.950-961.

Seol, D., Seo, J., Rhee, S.H., 2013. “Towed underwater PIV measurement for free-surface effects on turbulent wake of a surface-piercing body," International Journal of Naval Architecture and Ocean Engineering, Vol. 5, No. 3, pp. 404-413.

Toda, Y., Stern, F., Tanaka, I., Patel, V.C., 1990. "Mean-flow measurements in the boundary layer and wake of a series $60 \mathrm{C}_{\mathrm{B}}=0.60$ model ship with and without propeller," Journal of Ship Research, Vol. 34, No. 4, pp. 225-252.

Toda, Y., Stern, F., Longo, J., 1992. "Mean-flow measurements in the boundary layer and wake and wave field of a series $60 C_{B}=0.60$ ship model-part 1: Froude numbers 0.16 and 0.316," Journal of Ship Research, Vol. 36, No. 4, pp. 360-377.

Van, S., Kim, W., Yoon, H., Lee, Y., Park, I., 2006. "Flow measurement around a model ship with propeller and rudder," Experiments in Fluids, Vol. 40, No. 4, pp. 533-545.

Yang, J., Rhee, S.H., Kim, H., 2009. "Propulsive performance of a tanker hull form in damaged conditions," Ocean Engineering, Vol. 36, No. 2, pp. 133-144. 
Yoon, H., 2009. "Phase-averaged stereo-PIV flow field and force/moment/motion measurements for surface combatant in PMM maneuvers," Ph. D. Thesis, Department of Mechanical Engineering, The University of Iowa, Iowa City, IA. 


\section{LIST OF TABLES}

Table 1. Measurement uncertainties in a 2D PIV system.

Table 2. Principal dimensions of the KVLCC2 model.

Table 3. Principal dimensions of the generic tanker model. 
Table 1. Uncertainty of 2D PIV system in particle displacement

\begin{tabular}{|c|c|c|c|c|c|}
\hline & & Reference & Bias limit & Precision limit & Total uncertainty \\
\hline \multirow{3}{*}{$\begin{array}{l}\text { Mean velocity } \\
\text { measurement }\end{array}$} & $\overline{\boldsymbol{u}}$ & \multirow{3}{*}{$U$} & $1.03 \%$ & $0.54 \%$ & $1.16 \%$ \\
\hline & $\overline{\boldsymbol{v}}$ & & $1.72 \%$ & $0.62 \%$ & $1.83 \%$ \\
\hline & $\overline{\boldsymbol{w}}$ & & $1.64 \%$ & $0.65 \%$ & $1.76 \%$ \\
\hline \multirow{6}{*}{$\begin{array}{l}\text { Turbulence } \\
\text { properties }\end{array}$} & $\overline{\overline{\boldsymbol{u}^{\prime} \boldsymbol{u}^{\prime}}}$ & \multirow{6}{*}{$\begin{array}{l}U^{2} \\
/ 1000\end{array}$} & $10.4 \%$ & $7.9 \%$ & $13.0 \%$ \\
\hline & $\overline{\overline{v^{\prime} v^{\prime}}}$ & & $11.1 \%$ & $7.1 \%$ & $13.2 \%$ \\
\hline & $\overline{\overline{w^{\prime} w^{\prime}}}$ & & $12.5 \%$ & $8.3 \%$ & $15.0 \%$ \\
\hline & $\overline{\overline{u^{\prime} v^{\prime}}}$ & & $25.1 \%$ & $11.5 \%$ & $27.6 \%$ \\
\hline & $\overline{\overline{u^{\prime} w^{\prime}}}$ & & $21.0 \%$ & $18.2 \%$ & $27.8 \%$ \\
\hline & $k$ & & $35.2 \%$ & $12.4 \%$ & $37.3 \%$ \\
\hline
\end{tabular}


Table 2. Principal dimensions of the KVLCC2 model

\begin{tabular}{|c|c|c|c|}
\hline & & Full scale & Model \\
\hline Scale & & 1 & $1 / 100$ \\
\hline Length between perpendiculars & $\boldsymbol{L}_{\boldsymbol{P P}}$ & $320.0 \mathrm{~m}$ & $3.20 \mathrm{~m}$ \\
\hline Beam & $B$ & $58.0 \mathrm{~m}$ & $0.58 \mathrm{~m}$ \\
\hline Draft & $T$ & $20.8 \mathrm{~m}$ & $0.208 \mathrm{~m}$ \\
\hline Propeller diameter & $D$ & $9.8 \mathrm{~m}$ & $0.098 \mathrm{~m}$ \\
\hline Reynolds number based on $\boldsymbol{L}_{\boldsymbol{P P}}$ & $R \boldsymbol{R}$ & $2.14 \times 10^{9}$ & $2.32 \times 10^{6}$ \\
\hline Froude number based on $\boldsymbol{L}_{\boldsymbol{P P}}$ & $F r$ & \multicolumn{2}{|c|}{0.142} \\
\hline Block coefficient & $\boldsymbol{C}_{\boldsymbol{B}}$ & 0.8098 \\
\hline
\end{tabular}


Table 3. Principal dimensions of the generic tanker model

\begin{tabular}{|l|l|}
\hline & Model \\
\hline Length between perpendiculars & $3.20 \mathrm{~m}$ \\
\hline Beam & $0.60 \mathrm{~m}$ \\
\hline Draft & $0.21 \mathrm{~m}$ \\
\hline Propeller diameter & $0.098 \mathrm{~m}$ \\
\hline Reynolds number & $2.44 \times 10^{6}$ \\
\hline Froude number & 0.145 \\
\hline Block coefficient & 0.7936 \\
\hline
\end{tabular}




\section{LIST OF FIGURE CAPTIONS}

Figure 1. Schematic diagram of the towed underwater PIV system and test model.

Figure 2. 3D3C flow field reconstruction procedure (a: horizontal planes, b: vertical planes, c: interpolation, d: reconstructed volume).

Figure 3. Body plan and profiles of the KVLCC2.

Figure 4. Comparison of $\overline{\boldsymbol{u}_{\boldsymbol{h}}} / \boldsymbol{U}$ and $\overline{\boldsymbol{u}_{\boldsymbol{v}}} / \boldsymbol{U}$ from the nominal wake field of the KVLCC2 model: $\left(\overline{\boldsymbol{u}_{\boldsymbol{h}}}+\overline{\boldsymbol{u}_{\boldsymbol{v}}}\right) / \mathbf{2} \boldsymbol{U}$ (left), dashed line for $\overline{\boldsymbol{u}_{\boldsymbol{h}}} / \boldsymbol{U}$ and solid line for $\overline{\boldsymbol{u}_{\boldsymbol{v}}} / \boldsymbol{U}$ (right).

Figure 5. Comparison of $\overline{\boldsymbol{u}_{\boldsymbol{h}}} / \boldsymbol{U}$ and $\overline{\boldsymbol{u}_{\boldsymbol{v}}} / \boldsymbol{U}$ from the nominal wake field of the KVLCC2 model: $\overline{\boldsymbol{u}_{\boldsymbol{h}}} / \boldsymbol{U}$ and $\overline{\boldsymbol{u}_{\boldsymbol{v}}} / \boldsymbol{U}$ at constant vertical locations.

Figure 6. Nominal wake field of the KVLCC2 model: $\overline{\boldsymbol{u}} / \boldsymbol{U}$ Contours (left) and $\overline{\boldsymbol{v}} / \boldsymbol{U}-\overline{\boldsymbol{w}} / \boldsymbol{U}$ vectors (right).

Figure 7. Iso-surfaces of $\overline{\boldsymbol{u}} / \boldsymbol{U}=0.3,0.5$, and 0.7 from a flow field around the stern of the KVLCC2.

Figure 8. Nominal wake field of the KVLCC2 model: comparison of $\overline{\boldsymbol{u}} / \boldsymbol{U}$ at constant radii.

Figure 9. Nominal wake field of the KVLCC2 model: $\boldsymbol{\zeta}_{x} \boldsymbol{R} / \boldsymbol{U}$ contours (left) and stream lines (right).

Figure 10. Comparison of $\overline{\boldsymbol{u}^{\prime} \boldsymbol{u}_{h}^{\prime}} / \boldsymbol{U}^{2}$ and $\overline{\boldsymbol{u}^{\prime} \boldsymbol{u}^{\prime}{ }_{\boldsymbol{v}}} / \boldsymbol{U}^{2}$ from the nominal wake field of the KVLCC2 model: $\left(\overline{\boldsymbol{u}^{\prime} \boldsymbol{u}_{\boldsymbol{h}}^{\prime}}+\overline{\boldsymbol{u}^{\prime} \boldsymbol{u}_{\boldsymbol{v}}^{\prime}}\right) / \mathbf{2} \boldsymbol{U}^{\mathbf{2}} \times \mathbf{1 0 0 0}$ (left), dashed line for $\overline{\boldsymbol{u}^{\prime} \boldsymbol{u}_{\boldsymbol{h}}^{\prime}} / \boldsymbol{U}^{\mathbf{2}} \times$ 1000, and solid line for $\overline{\boldsymbol{u}^{\prime} \boldsymbol{u}_{v}^{\prime}} / U^{2} \times \mathbf{1 0 0 0}$ (right).

Figure 11. Comparison of $\overline{\boldsymbol{u}^{\prime} \boldsymbol{u}_{\boldsymbol{h}}^{\prime}} / \boldsymbol{U}^{2}$ and $\overline{\boldsymbol{u}^{\prime} \boldsymbol{u}^{\prime}}{ }_{\boldsymbol{v}} / \boldsymbol{U}^{\mathbf{2}}$ from the nominal wake field of the KVLCC2 model: $\overline{\boldsymbol{u}^{\prime} \boldsymbol{u}_{\boldsymbol{h}}^{\prime}} / \boldsymbol{U}^{2} \times \mathbf{1 0 0 0}$ and $\overline{\boldsymbol{u}^{\prime} \boldsymbol{u}_{\boldsymbol{v}}^{\prime}} / \boldsymbol{U}^{\mathbf{2}} \times \mathbf{1 0 0 0}$ at constant vertical locations. 
Figure 12. Nominal wake field of the KVLCC2 model: TKE and Reynolds stresses contours (A: $\boldsymbol{k} / \boldsymbol{U}^{2} \times 1000, \mathrm{~B}: \overline{\boldsymbol{u}^{\prime} \boldsymbol{u}^{\prime}} / \boldsymbol{U}^{2} \times 1000, \mathrm{C}: \overline{\boldsymbol{v}^{\prime} \boldsymbol{v}^{\prime}} / \boldsymbol{U}^{2} \times 1000, \mathrm{D}: \overline{\boldsymbol{w}^{\prime} \boldsymbol{w}^{\prime}} / \boldsymbol{U}^{2} \times 1000 \mathrm{E}$ : $\left.\overline{u^{\prime} v^{\prime}} / U^{2} \times 1000, \mathrm{~F}: \overline{u^{\prime} w^{\prime}} / U^{2} \times 1000\right)$

Figure 13. Nominal wake field of the KVLCC2 model: comparison of $\boldsymbol{k} / \boldsymbol{U}^{\mathbf{2}} \times \mathbf{1 0 0 0}$ at a constant radii.

Figure 14. Nominal wake field of the generic tanker model: $\overline{\boldsymbol{u}} / \boldsymbol{U}$ contours (left) and $\overline{\boldsymbol{v}} / \boldsymbol{U}-\overline{\boldsymbol{w}} / \boldsymbol{U}$ vectors (right).

Figure 15. Nominal wake field of the generic tanker model: TKE and Reynolds normal stress contours (A: $\left.\boldsymbol{k} / \boldsymbol{U}^{2} \times \mathbf{1 0 0 0}, \mathrm{B}: \overline{\boldsymbol{u}^{\prime} \boldsymbol{u}^{\prime}} / \boldsymbol{U}^{2} \times \mathbf{1 0 0 0}\right)$.

Figure 16. Locations of the measurement volume and sliced planes for the self-propulsion condition of the generic tanker model.

Figure 17. Phase-averaged horizontal wake plane of the generic tanker model in selfpropulsion condition $(z / R=0)$ : velocity vectors with the Galilean decomposition $\left(\boldsymbol{U}_{\boldsymbol{C}}=1.0\right)$ and locations of tip vortices (indicated by arrows).

Figure 18. Phase-averaged horizontal wake plane of the generic tanker model in selfpropulsion condition $(z / R=0): \overline{\boldsymbol{u}_{\boldsymbol{h}}} / \boldsymbol{U}$ contours.

Figure 19. Estimation of propeller inflow at $r / R=0.7$ of the generic tanker model in selfpropulsion condition: the angle of attack onto the blade (top) and inflow velocity magnitude non-dimensionalized by $U$ (bottom).

Figure 20. $\overline{\boldsymbol{u}_{\boldsymbol{h}}} / \boldsymbol{U}$ contours on the horizontal plane $(z / R=0)$ of the generic tanker model in self-propulsion condition.

Figure 21. $\overline{\boldsymbol{u}_{\boldsymbol{h}}} / \boldsymbol{U}$ distributions of a horizontal wake field of the generic tanker model in selfpropulsion condition $(z / R=0)$. 
Figure 22. Phase-averaged horizontal wake plane of the generic tanker model in selfpropulsion condition $(z / R=0): \zeta_{x} R / \boldsymbol{U}$ contours and locations of tip vortices (indicated by arrows).

Figure 23. Phase-averaged vertical wake plane of the generic tanker model in self-propulsion condition: magnitude of $\zeta_{y} R / U$ contours.

Figure 24. Phase-averaged wake field of the generic tanker model: tip vortex trajectory identified by the iso-surfaces of helicity.

Figure 25. Phase-averaged wake field of the generic tanker model in self-propulsion condition: $\overline{\boldsymbol{u}} / \boldsymbol{U}$ contours and $\overline{\boldsymbol{v}} / \boldsymbol{U}-\overline{\boldsymbol{w}} / \boldsymbol{U}$ vectors (A: station 0.23 , B: station 0.16 , and C: station 0).

Figure 26. Phase-averaged wake field of the generic tanker model in self-propulsion condition: $\boldsymbol{k} / \boldsymbol{U}^{2} \times \mathbf{1 0 0 0}$ contours (A: station 0.23 , B: station 0.16 , and C: station 0 ). 


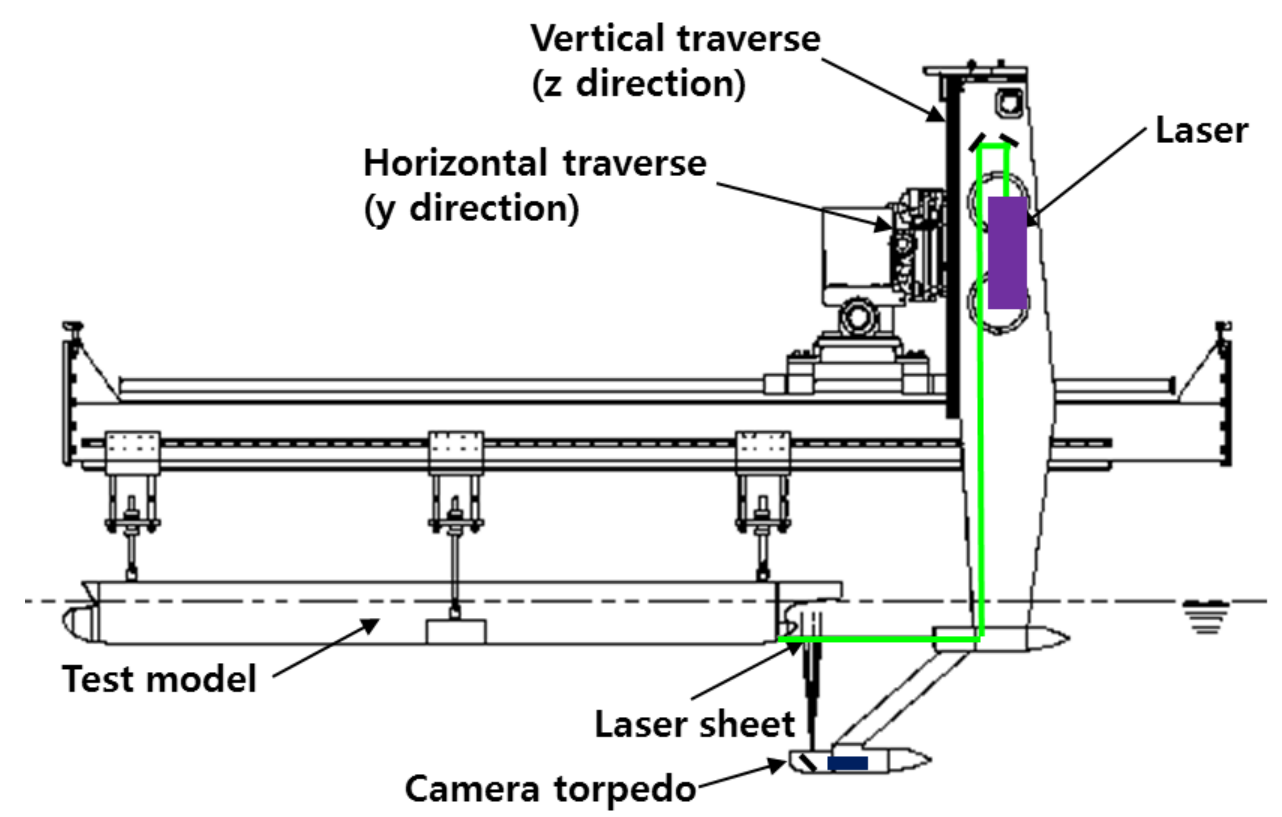

Figure 1. Schematic diagram of the towed underwater PIV system and test model. 


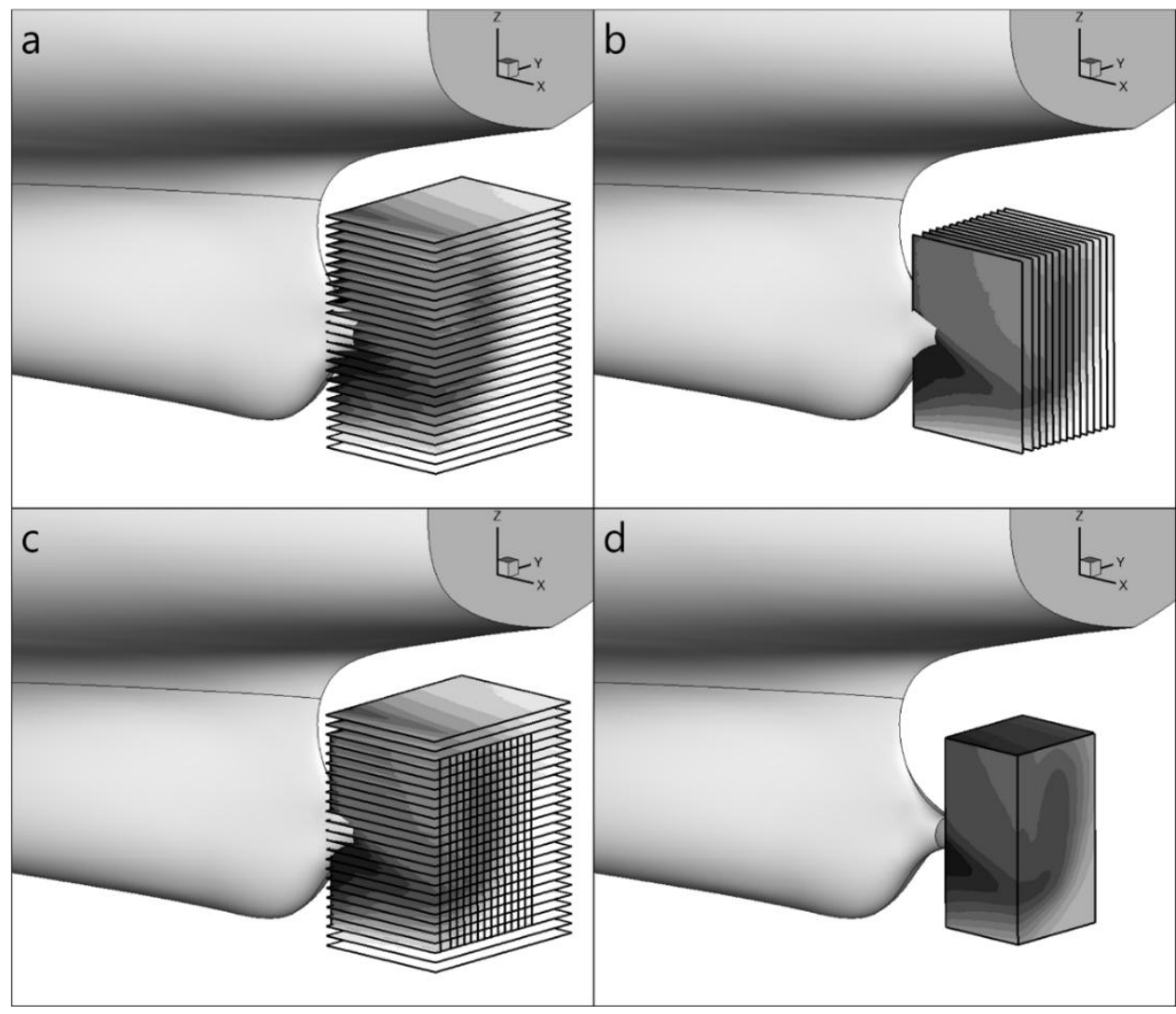

Figure 2. 3D3C flow field reconstruction procedure (a: horizontal planes, b: vertical planes, c: interpolation, $\mathrm{d}$ : reconstructed volume). 
1

2

3

4

5

6

7

9

10

11

12

13

14

15

16

17

18

19

20

21

22

23

24

25

26

27

28

29

30

31

32

33

34

35

36

37

38

39

40

41

42

43

44

45

46

47

48

49

50

51

52

53

54

55

56

57

58

59

60

61

62

63

64

65
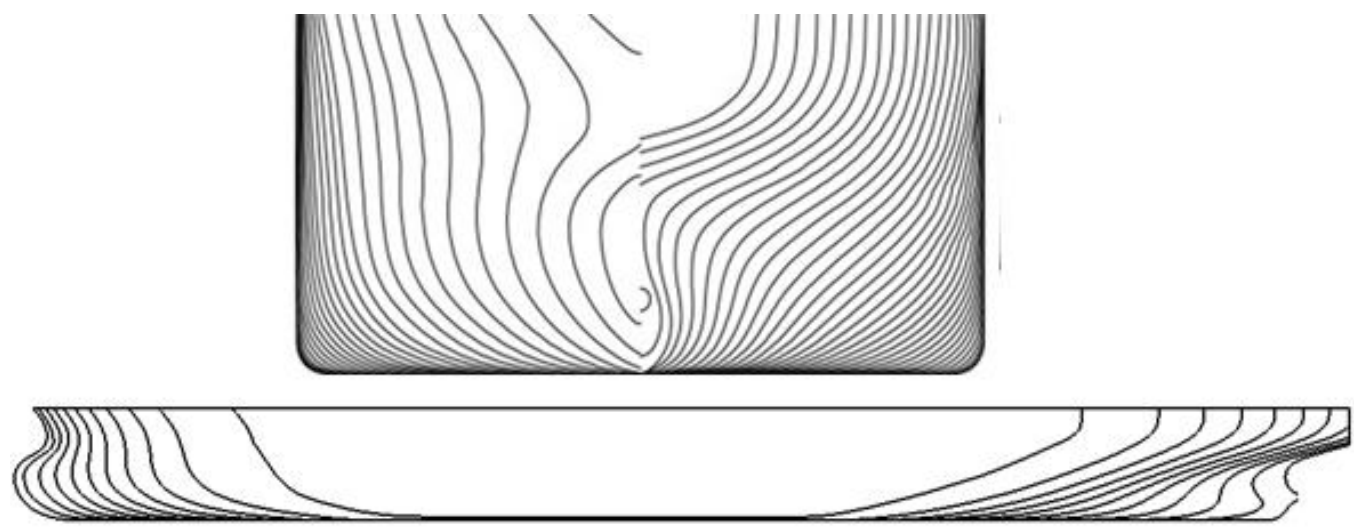

Figure 3. Body plan and profiles of the KVLCC2. 


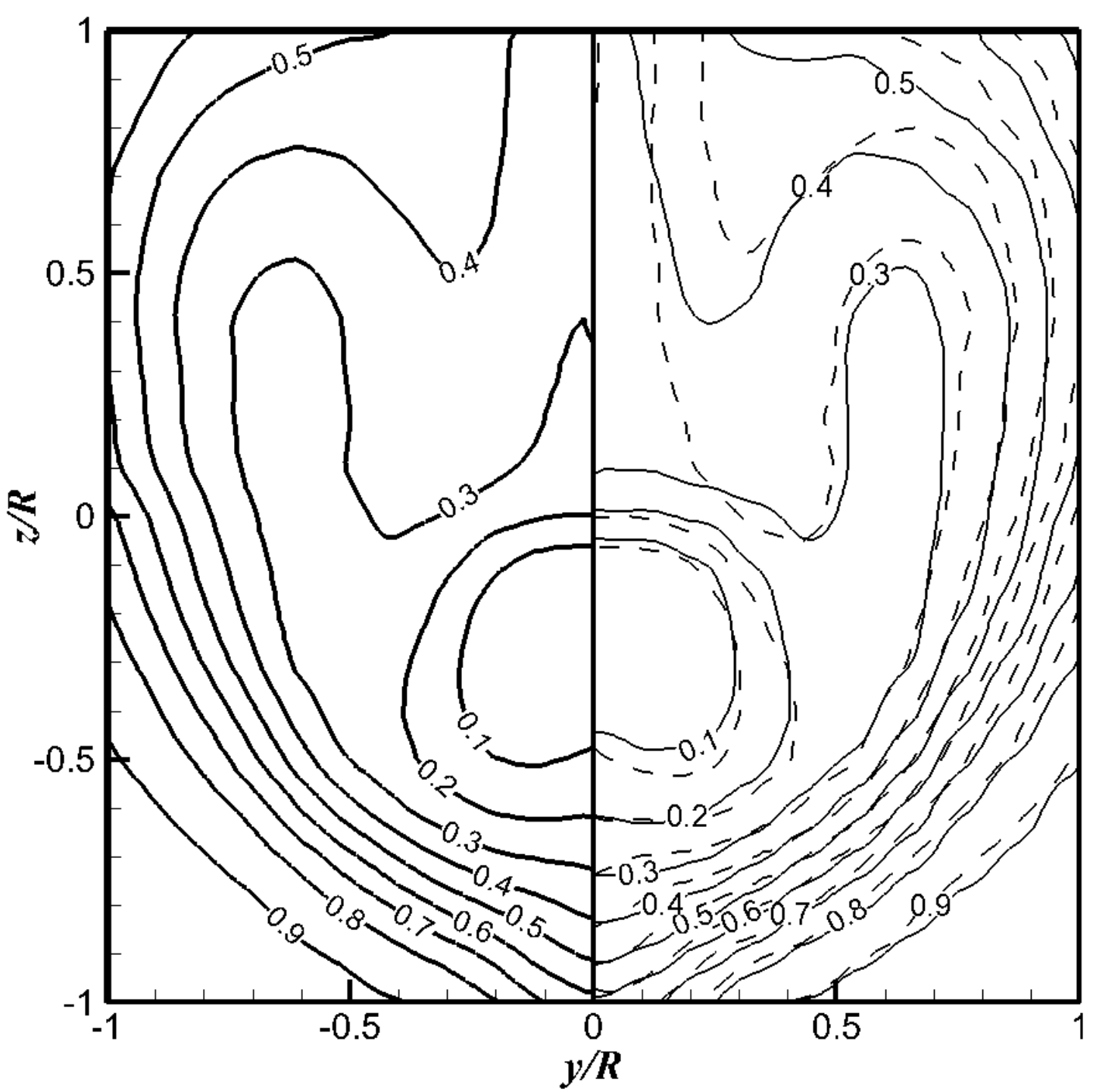

Figure 4. Comparison of $\overline{\boldsymbol{u}_{\boldsymbol{h}}} / \boldsymbol{U}$ and $\overline{\boldsymbol{u}_{\boldsymbol{v}}} / \boldsymbol{U}$ from the nominal wake field of the KVLCC2 model: $\left(\overline{\boldsymbol{u}_{\boldsymbol{h}}}+\overline{\boldsymbol{u}_{v}}\right) / \mathbf{2} \boldsymbol{U}$ (left), dashed line for $\overline{\boldsymbol{u}_{\boldsymbol{h}}} / \boldsymbol{U}$ and solid line for $\overline{\boldsymbol{u}_{\boldsymbol{v}}} / \boldsymbol{U}$ (right). 


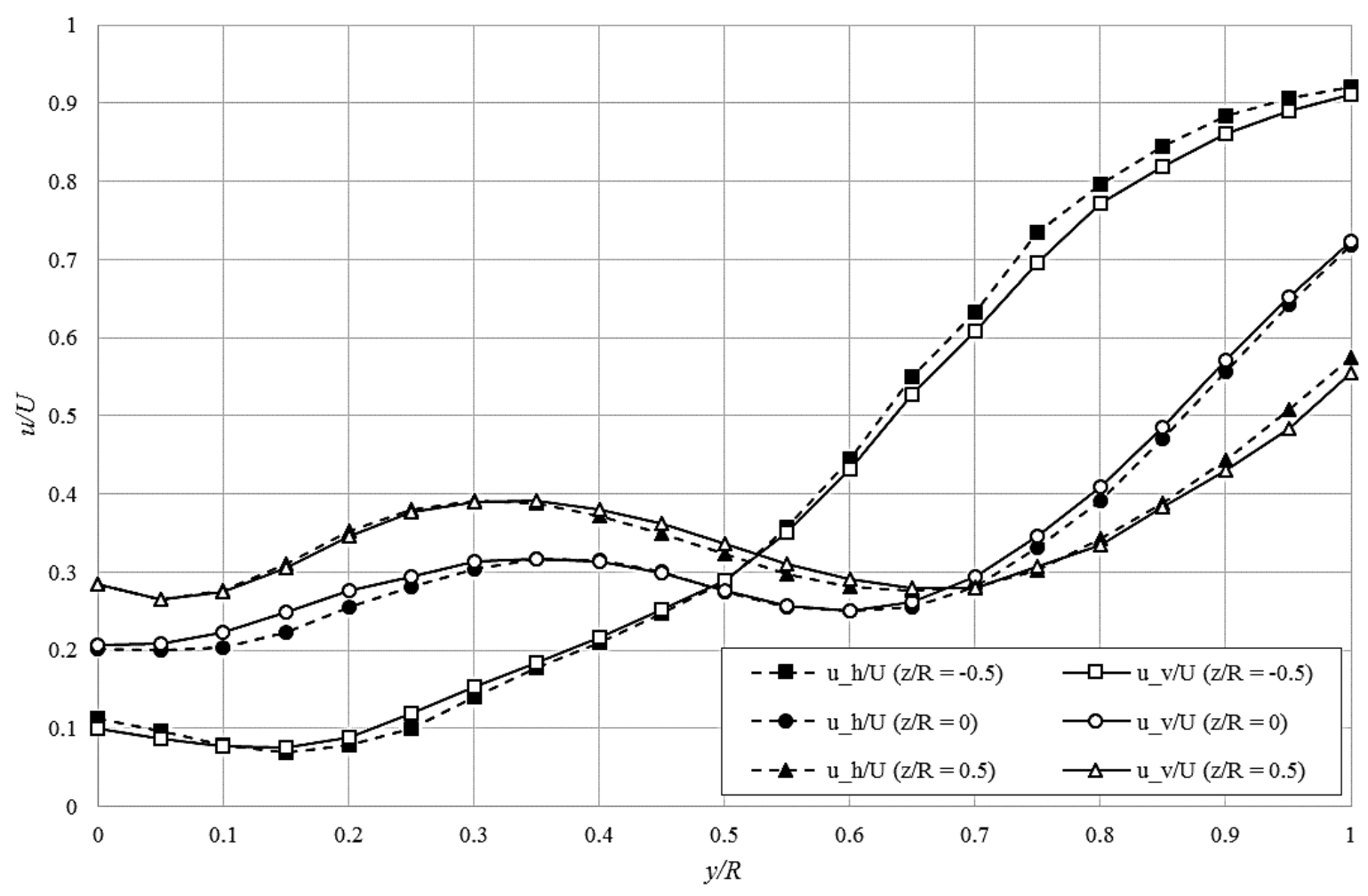

Figure 5. Comparison of $\overline{\boldsymbol{u}_{\boldsymbol{h}}} / \boldsymbol{U}$ and $\overline{\boldsymbol{u}_{\boldsymbol{v}}} / \boldsymbol{U}$ from the nominal wake field of the KVLCC2 model: $\overline{\boldsymbol{u}_{\boldsymbol{h}}} / \boldsymbol{U}$ and $\overline{\boldsymbol{u}_{\boldsymbol{v}}} / \boldsymbol{U}$ at constant vertical locations. 
1

2

3

4

5

6

7

8

9
10

11

12

13

14

15

16

17

18

19

20

21

22

23

24

25

26

27

28

29

30

31

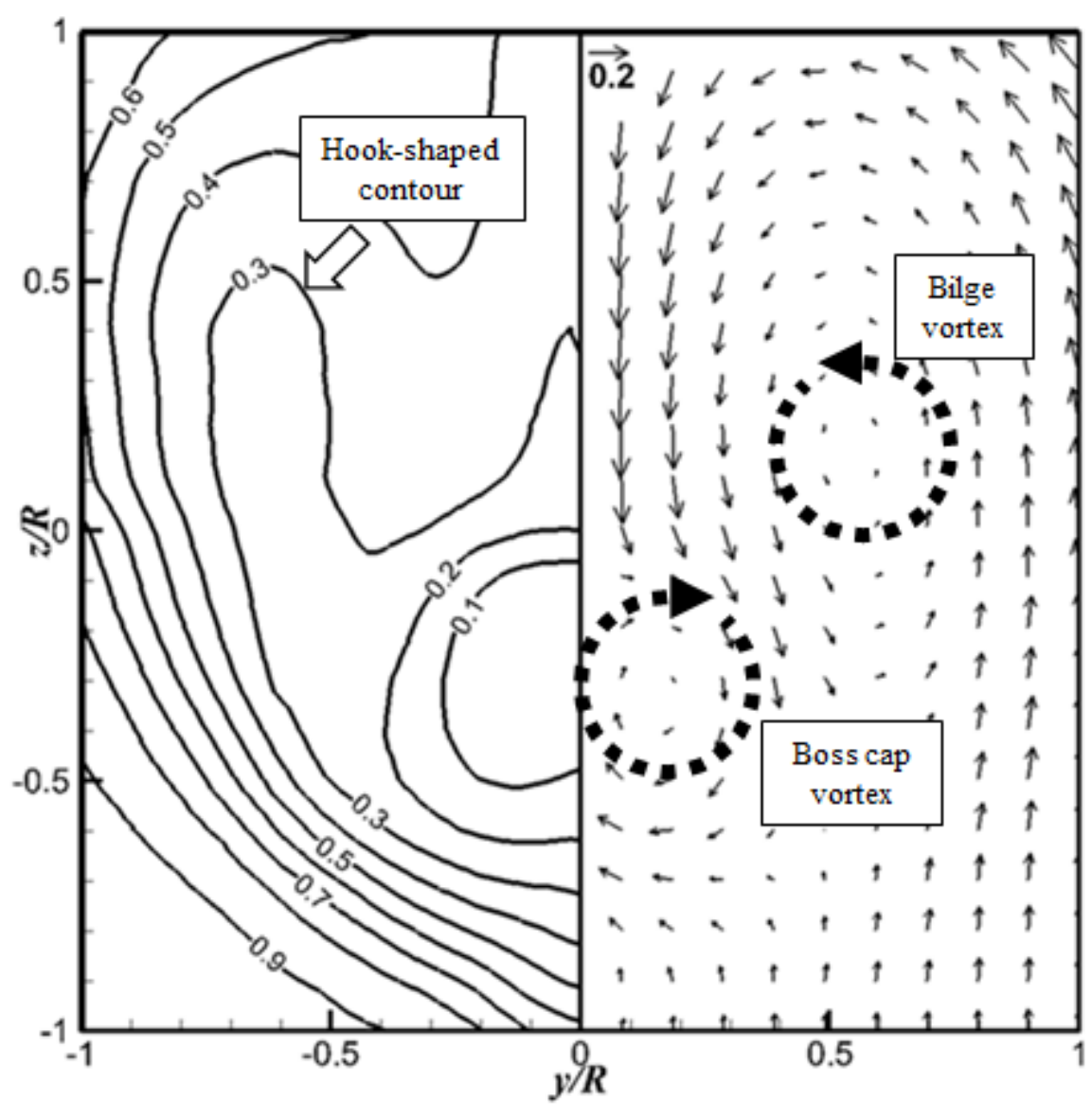

Figure 6. Nominal wake field of the KVLCC2 model: $\overline{\boldsymbol{u}} / \boldsymbol{U}$ Contours (left) and $\overline{\boldsymbol{v}} / \boldsymbol{U}-\overline{\boldsymbol{w}} / \boldsymbol{U}$ vectors (right). 


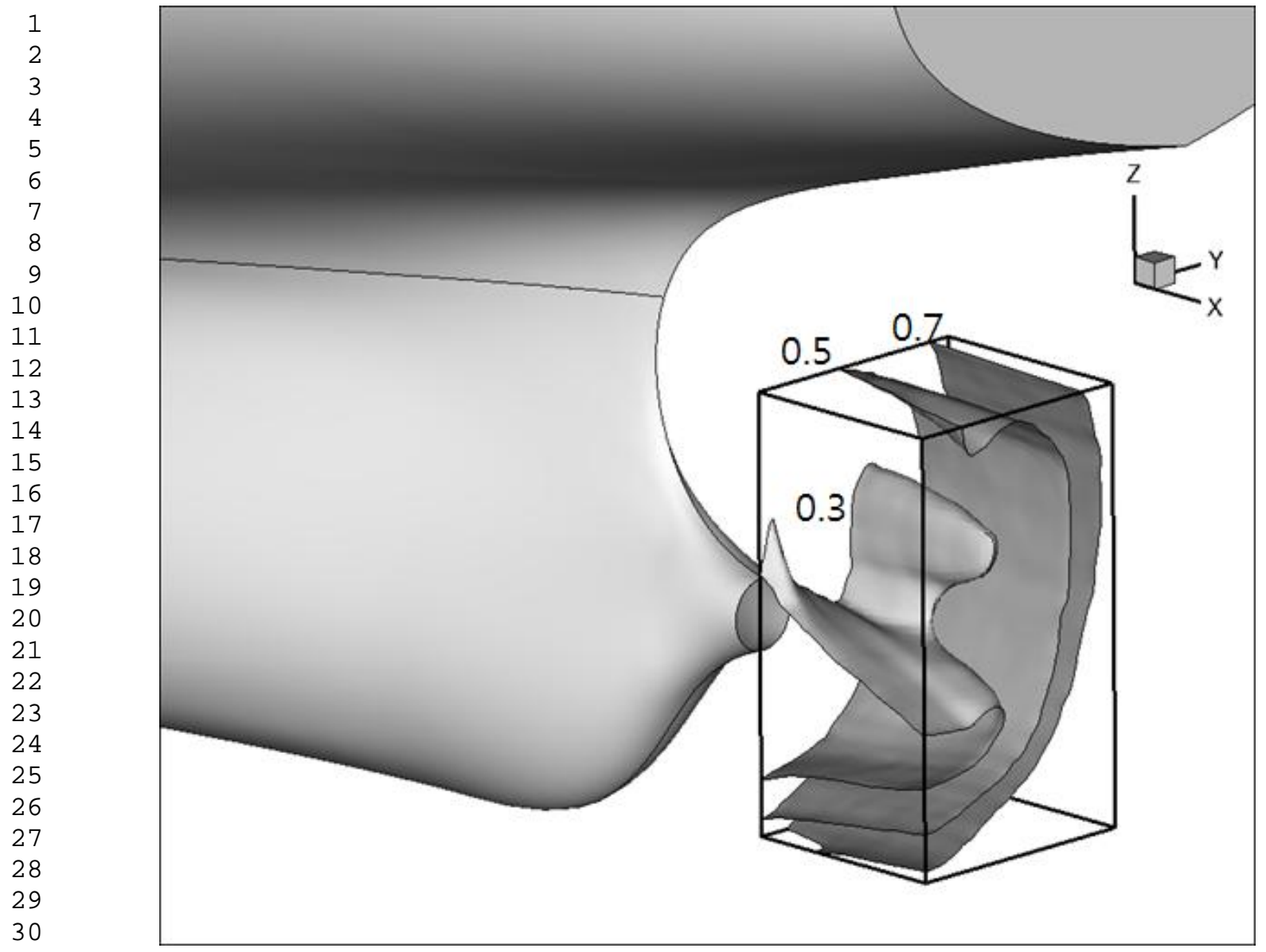

Figure 7. Iso-surfaces of $\overline{\boldsymbol{u}} / \boldsymbol{U}=0.3,0.5$, and 0.7 from a flow field around the stern of the KVLCC2. 


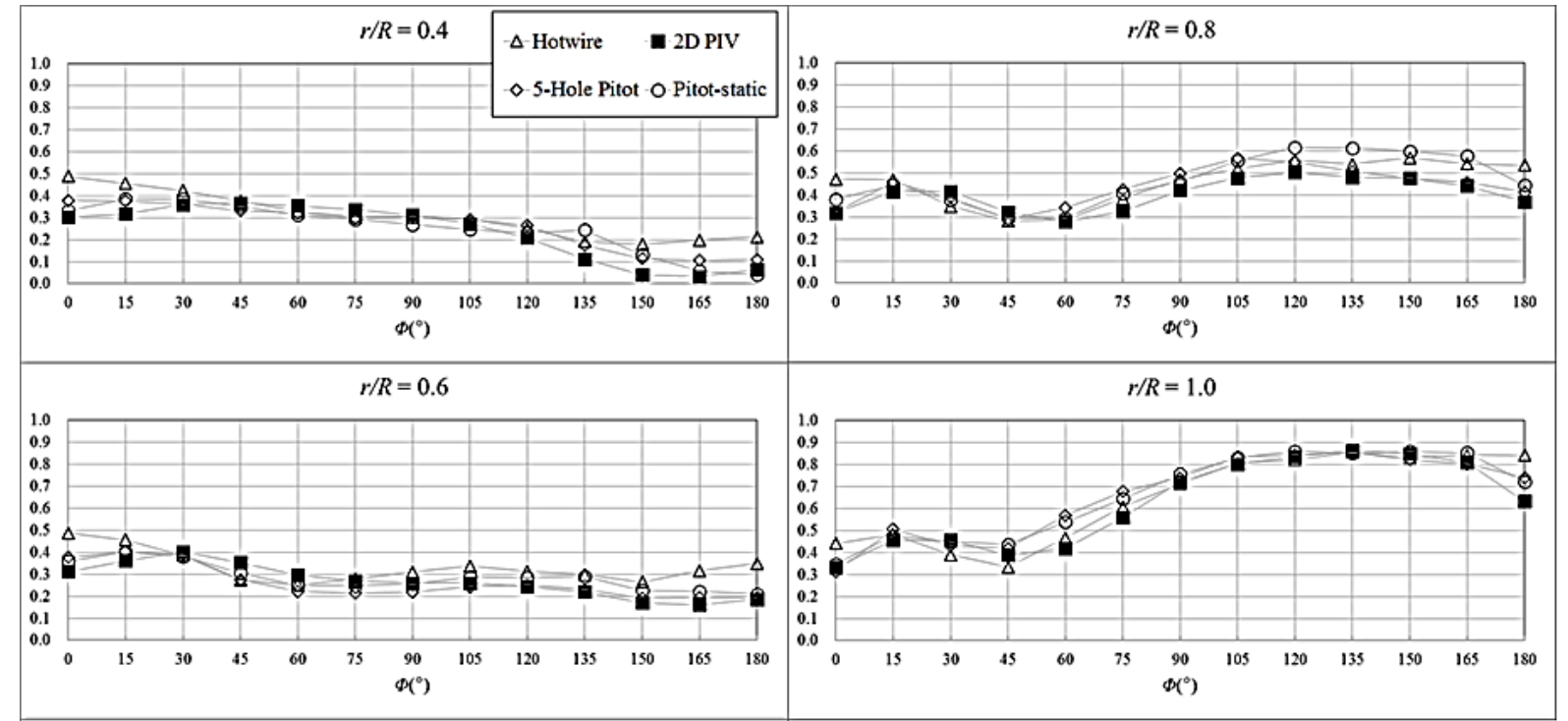

Figure 8. Nominal wake field of the KVLCC2 model: comparison of $\overline{\boldsymbol{u}} / \boldsymbol{U}$ at constant radii. 
1

2

3

4

5

6

7

8

9

10

11

12

13

14

15

16

17

18

19

20

21

22

23

24

25

26

27

28

29

30

31

32

33

34

35

36

37

38

39

40

41

42

43

44

45

46

47

48

49

50

51

52

53

54

55

56

57

58

59

60

61

62

63

64

65

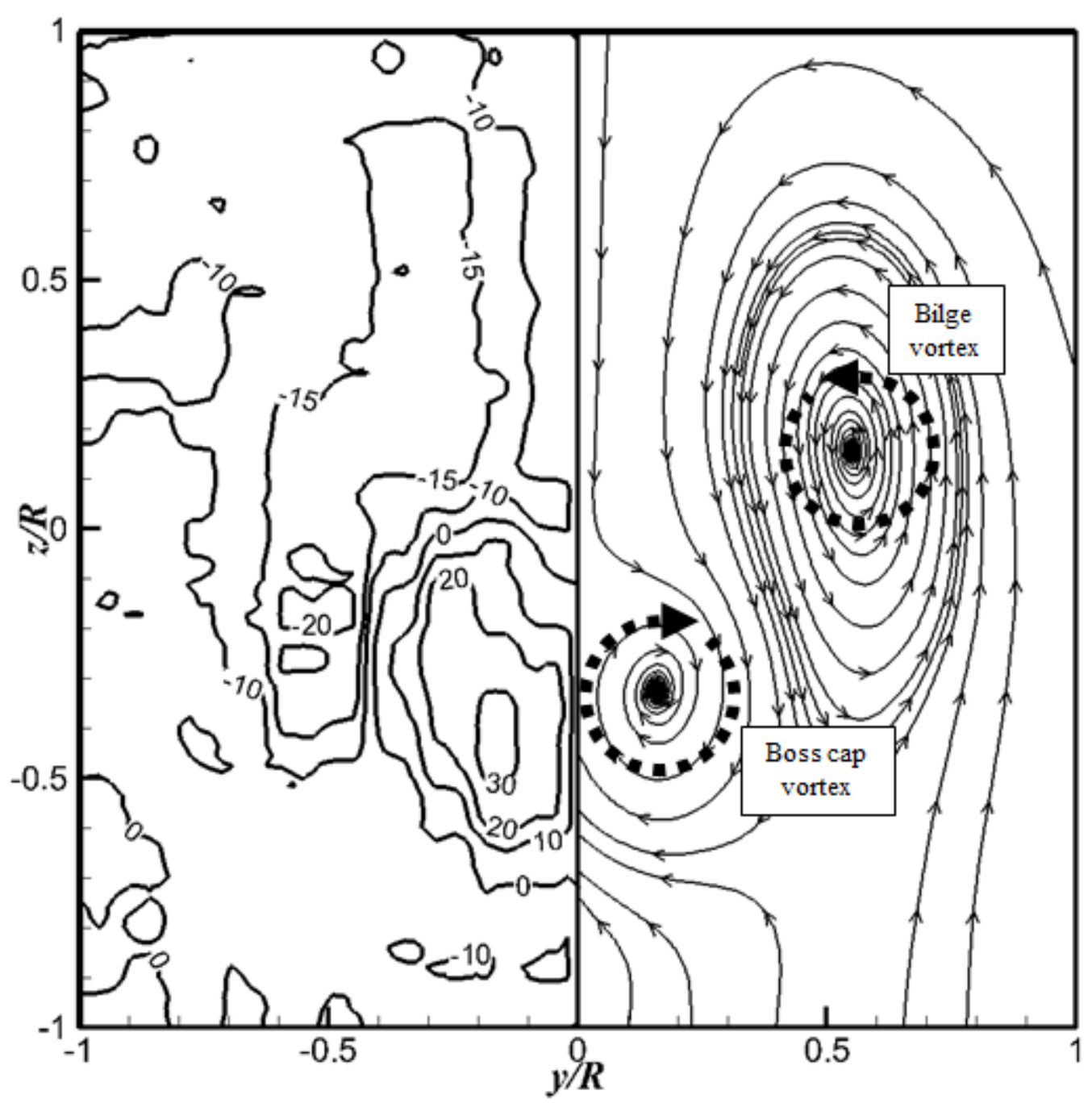

Figure 9. Nominal wake field of the KVLCC2 model: $\boldsymbol{\zeta}_{x} \boldsymbol{R} / \boldsymbol{U}$ contours (left) and stream lines (right). 


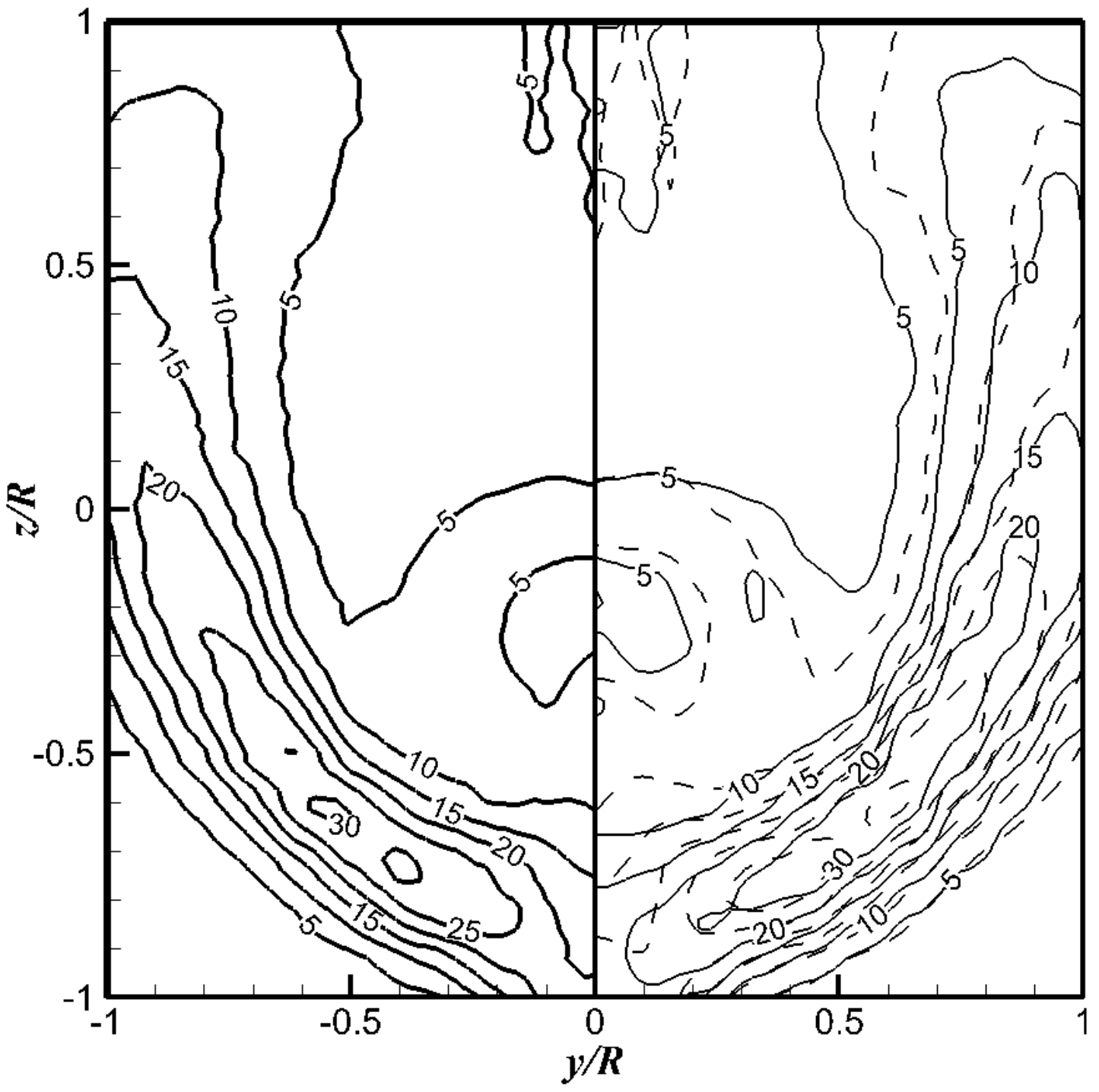

Figure 10. Comparison of $\overline{\boldsymbol{u}^{\prime} \boldsymbol{u}_{\boldsymbol{h}}^{\prime}} / \boldsymbol{U}^{\mathbf{2}}$ and $\overline{\boldsymbol{u}^{\prime} \boldsymbol{u}^{\prime}{ }_{\boldsymbol{v}}} / \boldsymbol{U}^{\mathbf{2}}$ from the nominal wake field of the KVLCC2 model: $\left(\overline{\boldsymbol{u}^{\prime} \boldsymbol{u}_{h}^{\prime}}+\overline{\boldsymbol{u}^{\prime} \boldsymbol{u}_{v}^{\prime}}\right) / \mathbf{2} \boldsymbol{U}^{\mathbf{2}} \times \mathbf{1 0 0 0}$ (left), dashed line for $\overline{\boldsymbol{u}^{\prime} \boldsymbol{u}_{h}^{\prime}} / \boldsymbol{U}^{2} \times$ 1000, and solid line for $\overline{\boldsymbol{u}^{\prime} \boldsymbol{u}_{v}^{\prime}} / \boldsymbol{U}^{2} \times \mathbf{1 0 0 0}$ (right). 


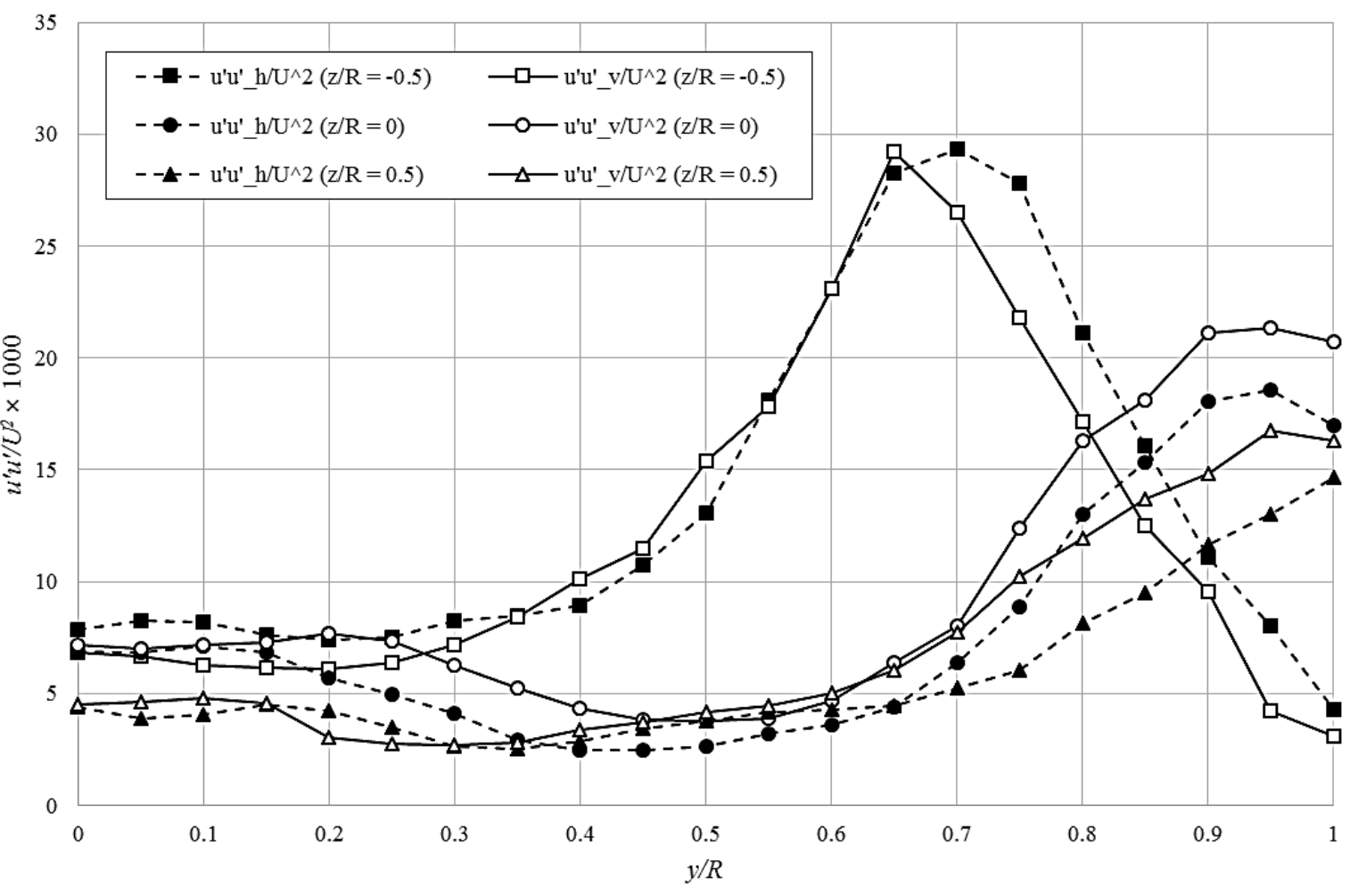

Figure 11. Comparison of $\overline{\boldsymbol{u}^{\prime} \boldsymbol{u}_{\boldsymbol{h}}^{\prime}} / \boldsymbol{U}^{2}$ and $\overline{\boldsymbol{u}^{\prime} \boldsymbol{u}_{\boldsymbol{v}}^{\prime}} / \boldsymbol{U}^{2}$ from the nominal wake field of the KVLCC2 model: $\overline{\boldsymbol{u}^{\prime} \boldsymbol{u}_{\boldsymbol{h}}^{\prime}} / \boldsymbol{U}^{2} \times \mathbf{1 0 0 0}$ and $\overline{\boldsymbol{u}^{\prime} \boldsymbol{u}_{v}^{\prime}} / \boldsymbol{U}^{2} \times \mathbf{1 0 0 0}$ at constant vertical locations. 


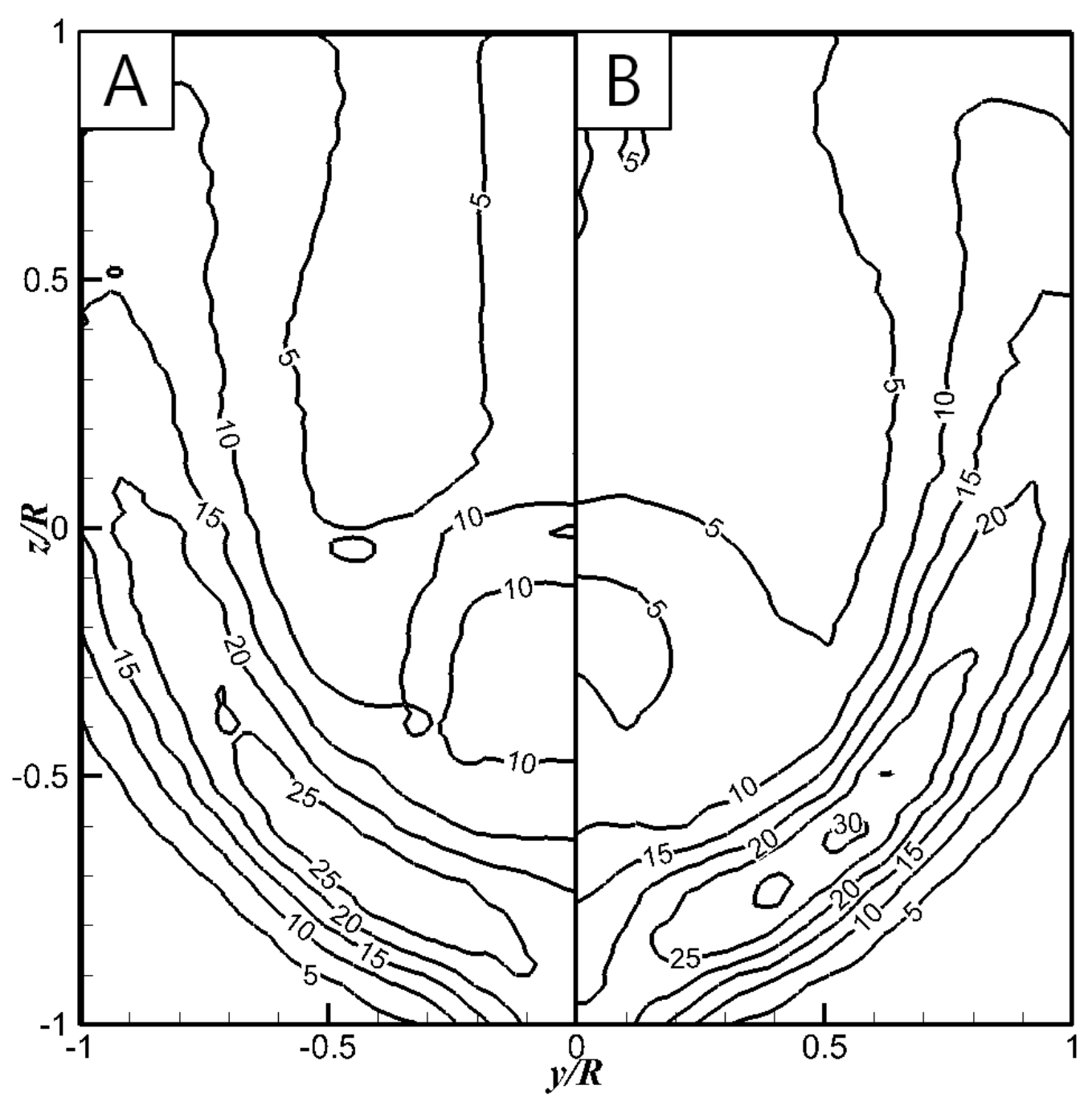




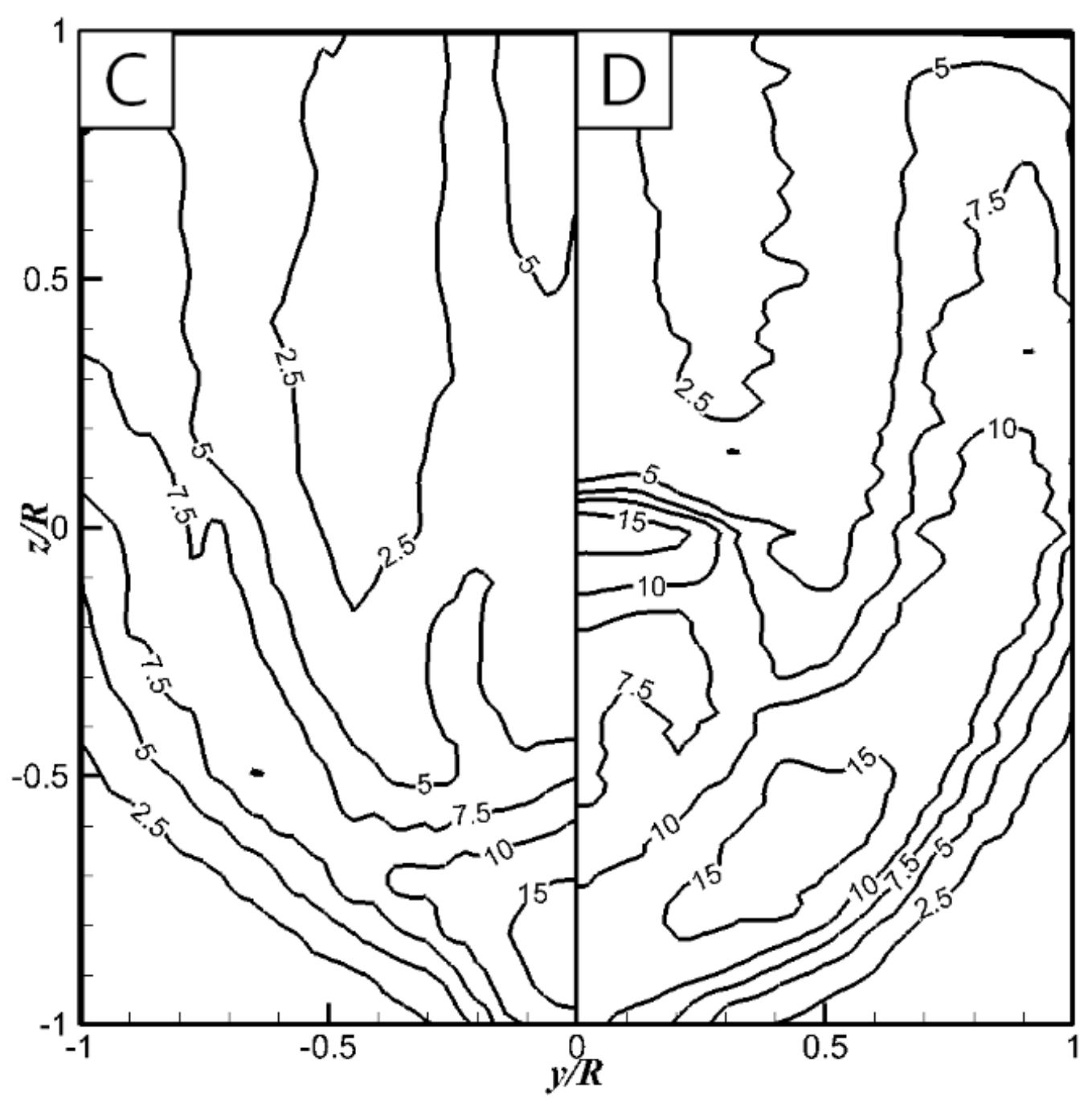


1

2

3

4

5

6

7

8

9
10

11

12

13

14

15

16

17

18

19

20

21

22

23

24

25

26

27

28

29

30

31

32

33

34

35

36

37

38

39

40

41

42

43

44

45

46

47

48

49

50

51

52

53

54

55

56

57

58

59

60

61

62

63

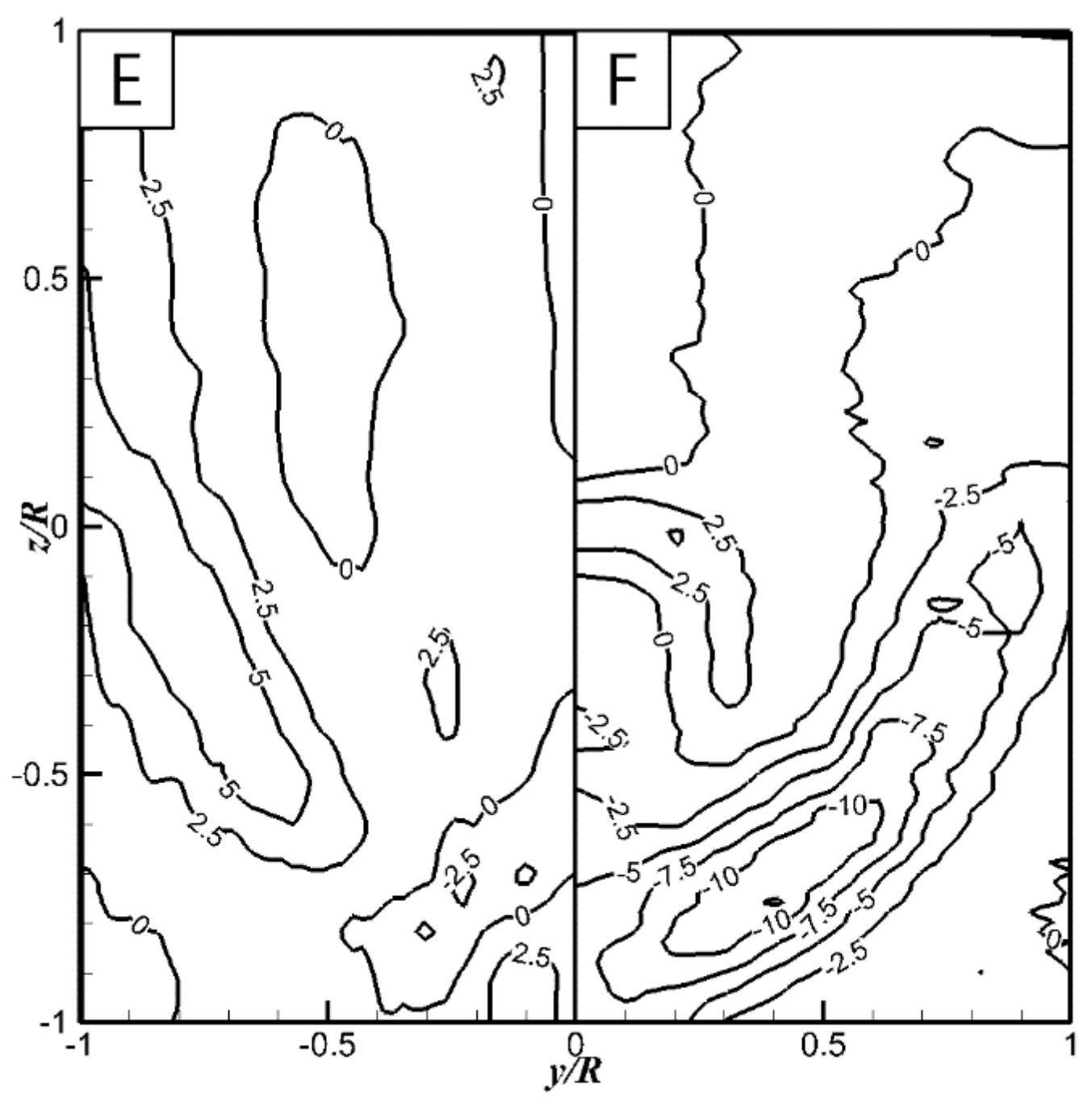

Figure 12. Nominal wake field of the KVLCC2 model: TKE and Reynolds stresses contours (A: $k / U^{2} \times 1000$, B: $\overline{u^{\prime} u^{\prime}} / U^{2} \times 1000$, C: $\overline{v^{\prime} v^{\prime}} / U^{2} \times 1000$, D: $\overline{w^{\prime} w^{\prime}} / U^{2} \times 1000 \mathrm{E}$ : $\left.\overline{u^{\prime} v^{\prime}} / U^{2} \times 1000, \mathrm{~F}: \overline{u^{\prime} w^{\prime}} / U^{2} \times 1000\right)$ 


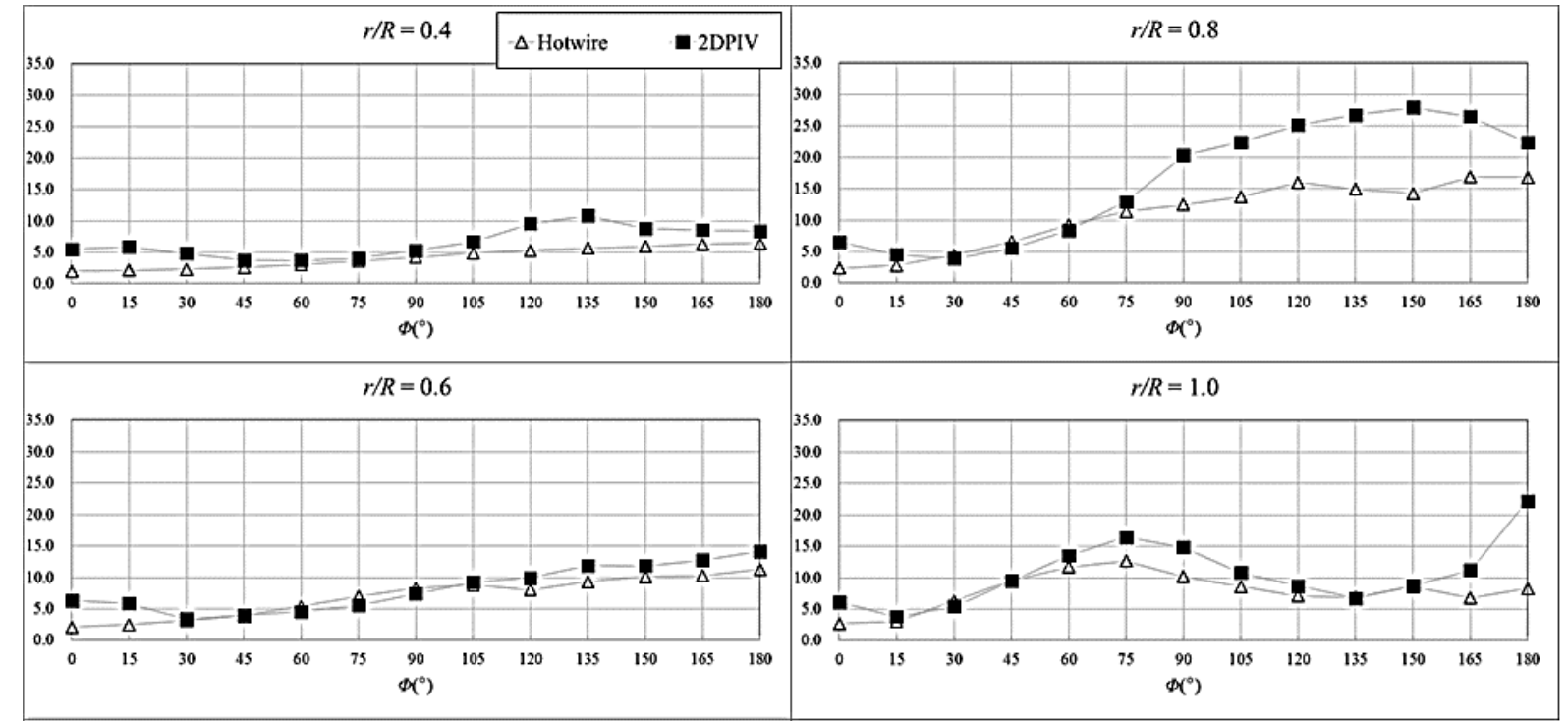

Figure 13. Nominal wake field of the KVLCC2 model: comparison of $\boldsymbol{k} / \boldsymbol{U}^{\mathbf{2}} \times \mathbf{1 0 0 0}$ at a constant radii. 
1

2

3

4

5

6

7

8

10

11

12

13

14

15

16

17

18

19

20

21

22

23

24

25

26

27

28

29

30

31

32

33

34

35

36

37

38

39

40

41

42

43

44

45

46

47

48

49

50

51

52

53

54

55

56

57

58

59

60

61

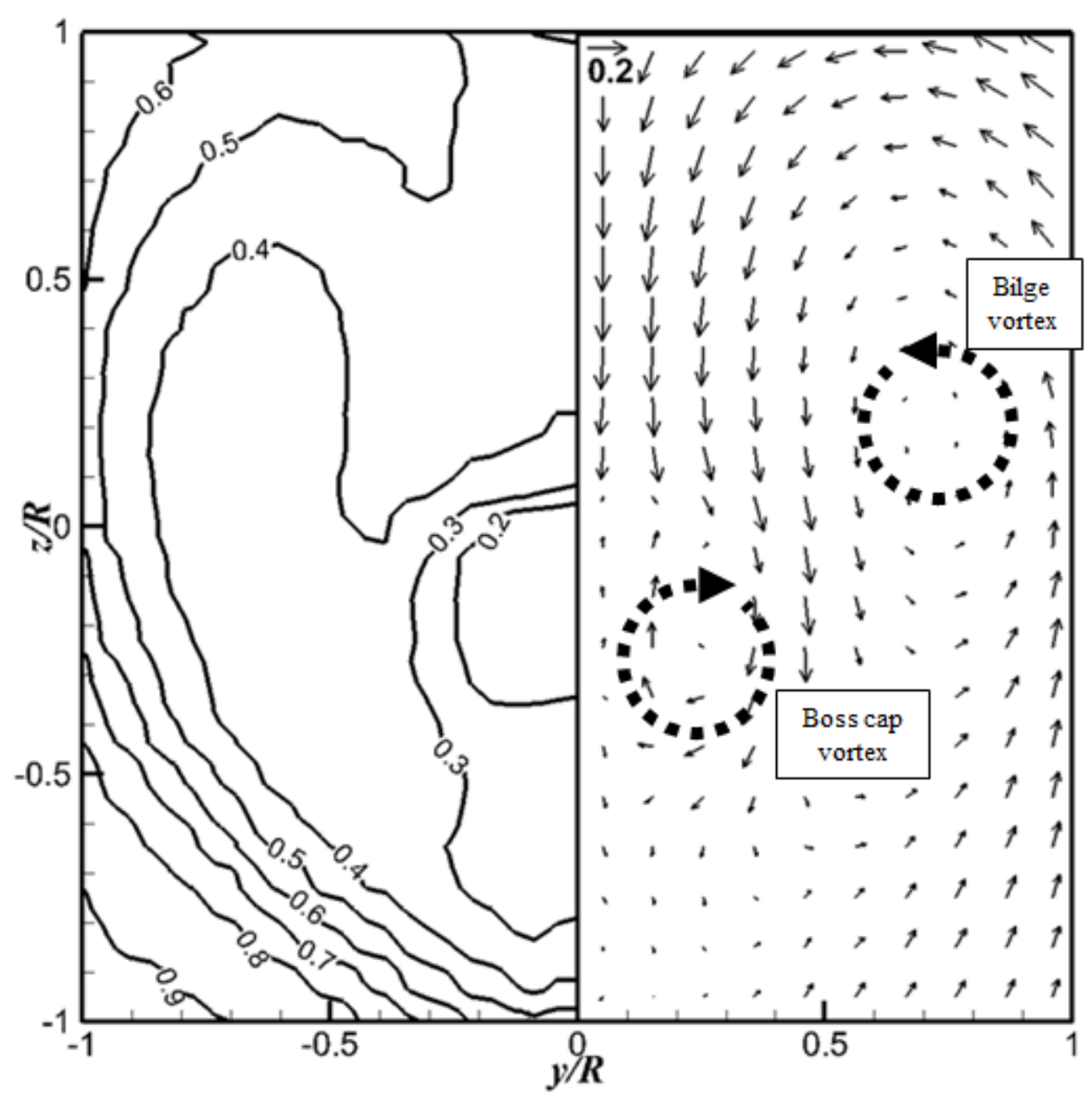

Figure 14. Nominal wake field of the generic tanker model: $\overline{\boldsymbol{u}} / \boldsymbol{U}$ contours (left) and $\overline{\boldsymbol{v}} / \boldsymbol{U}-\overline{\boldsymbol{w}} / \boldsymbol{U}$ vectors (right). 
1

2

3

4

5

6

7

8

9
10

11

12

13

14

15

16

17

18

19

20

21

22

23

24

25

26

27

28

29

30

31

32

33

34

35

36

37

38

39

40

41

42

43

44

45

46

47

48

49

50

51

52

53

54

55

56

57

58

59

60

61

62

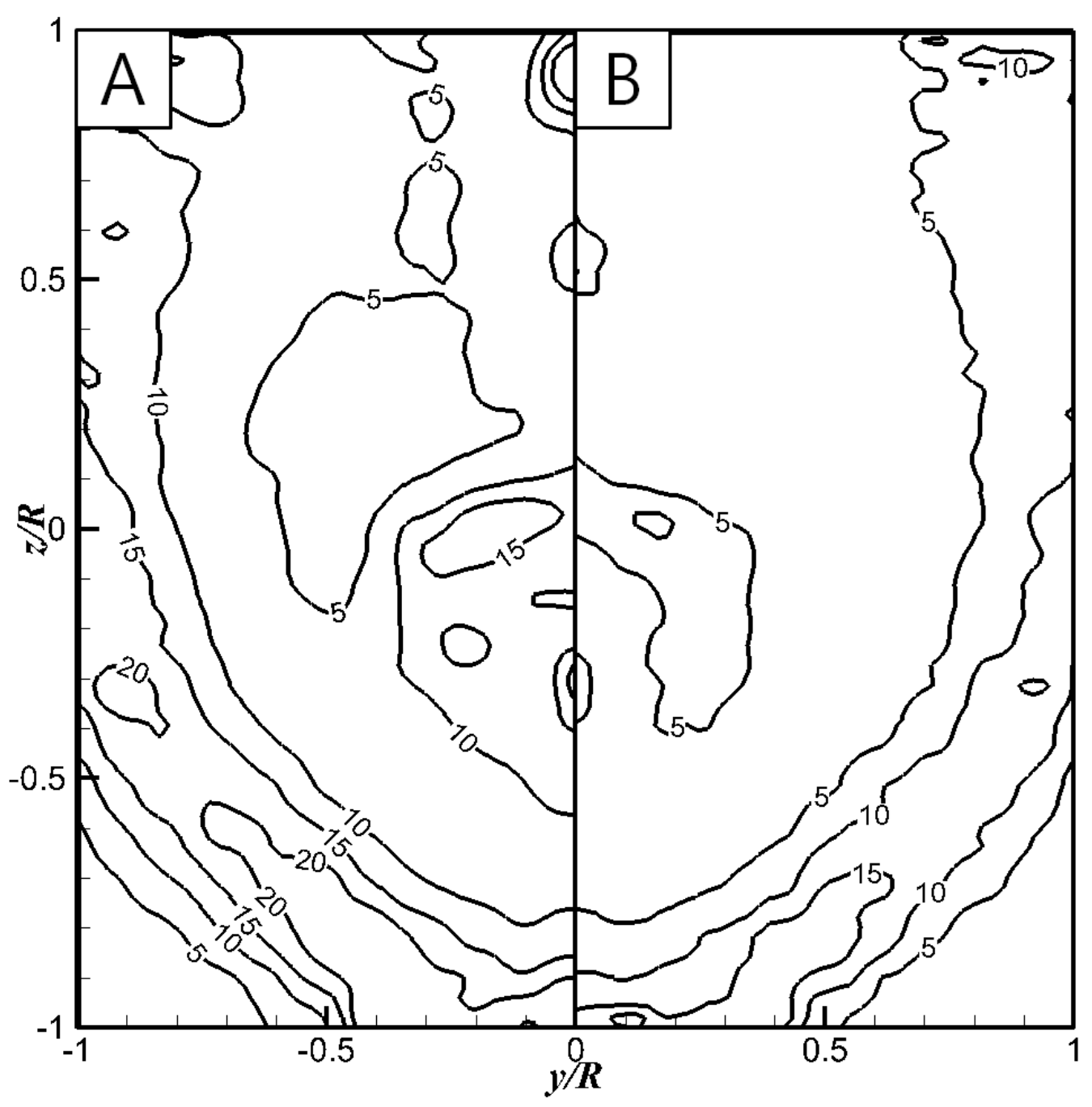

Figure 15. Nominal wake field of the generic tanker model: TKE and Reynolds normal stress contours (A: $\boldsymbol{k} / \boldsymbol{U}^{2} \times \mathbf{1 0 0 0}, \mathrm{B}: \overline{\boldsymbol{u}^{\prime} \boldsymbol{u}^{\prime}} / \boldsymbol{U}^{2} \times \mathbf{1 0 0 0}$ ). 


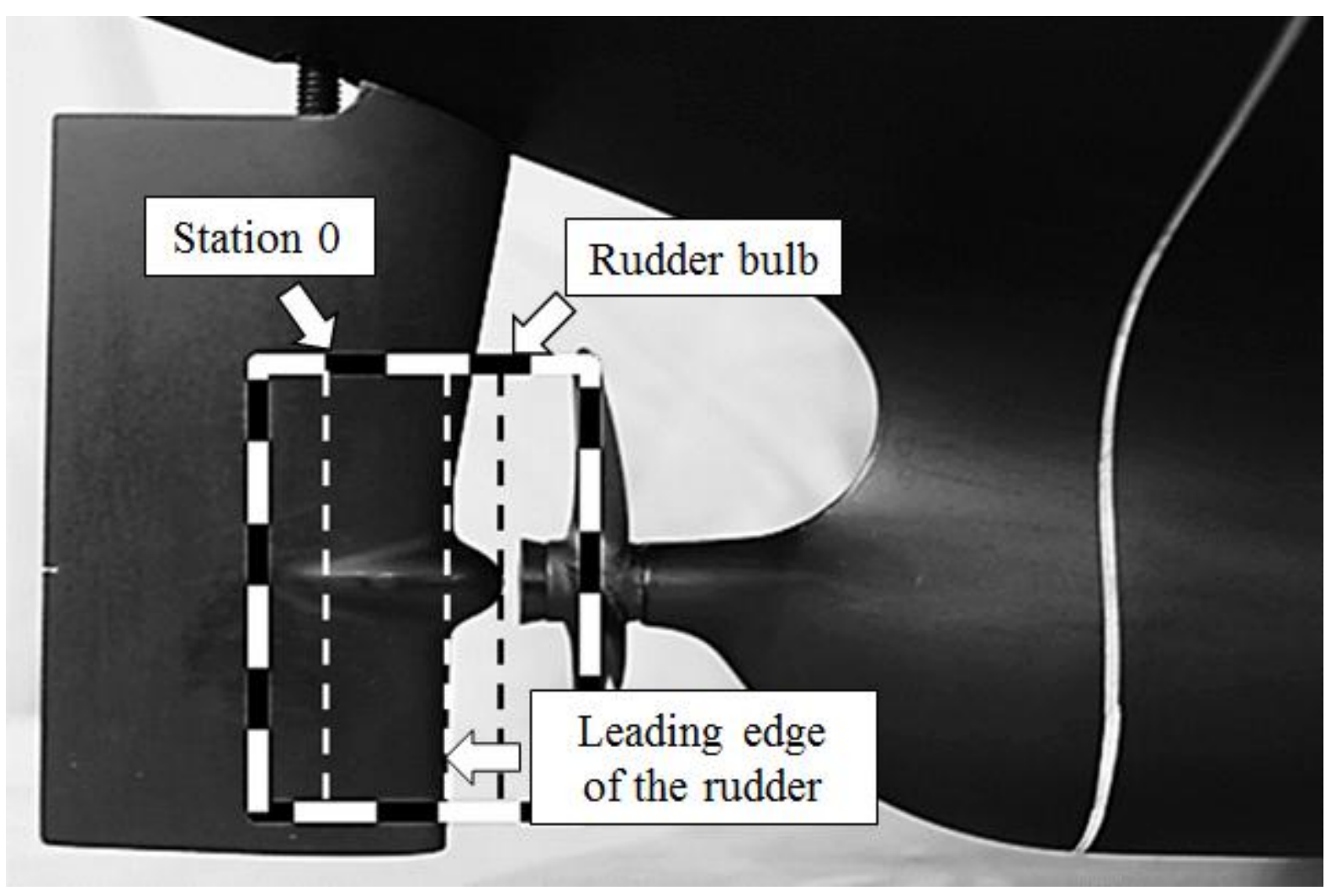

Figure 16. Locations of the measurement volume and sliced planes for the self-propulsion condition of the generic tanker model. 


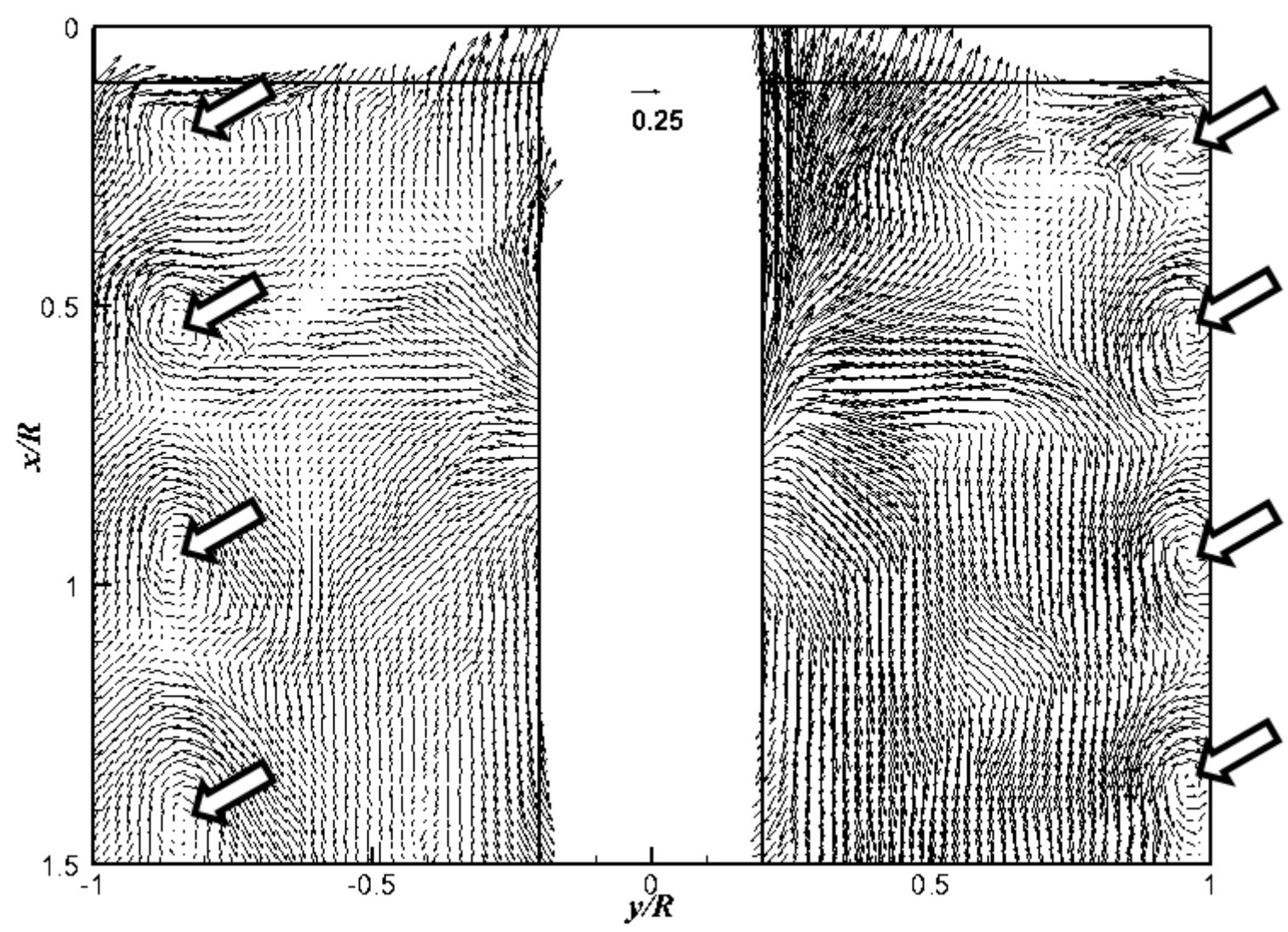

Figure 17. Phase-averaged horizontal wake plane of the generic tanker model in selfpropulsion condition $(z / R=0)$ : velocity vectors with the Galilean decomposition $\left(\boldsymbol{U}_{\boldsymbol{C}}=1.0\right)$ and locations of tip vortices (indicated by arrows). 


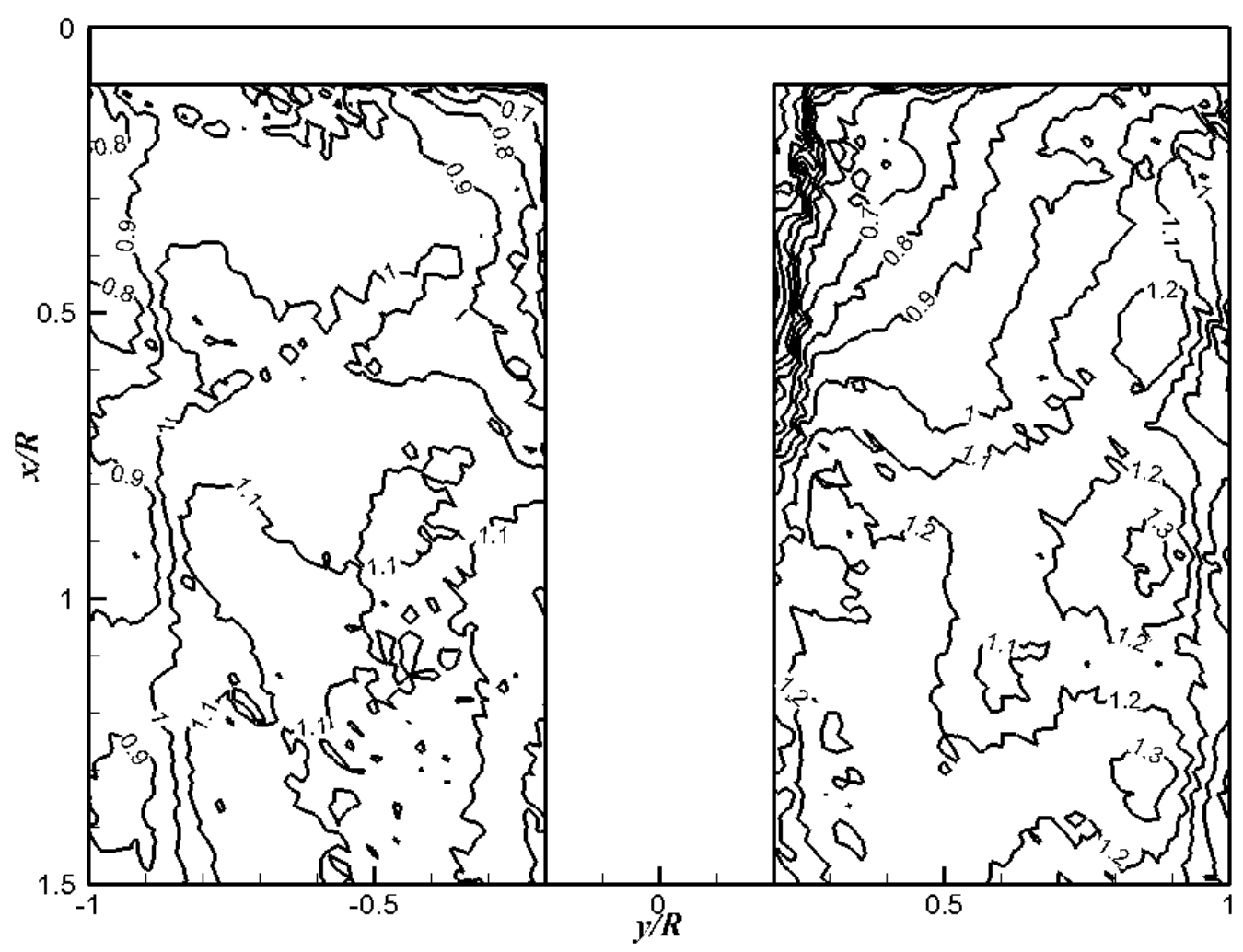

Figure 18. Phase-averaged horizontal wake plane of the generic tanker model in selfpropulsion condition $(z / R=0): \overline{\boldsymbol{u}_{\boldsymbol{h}}} / \boldsymbol{U}$ contours. 

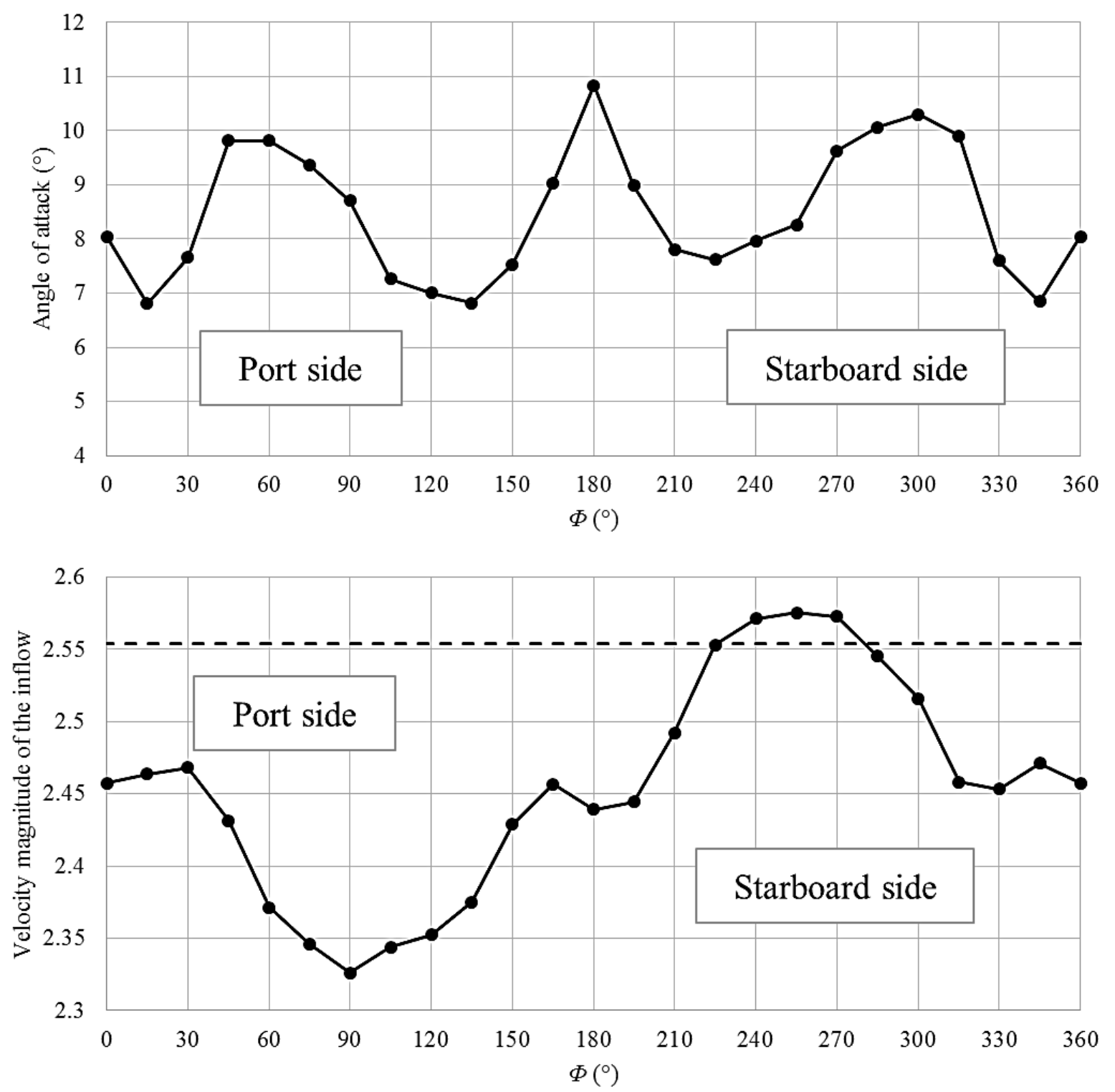

Figure 19. Estimation of propeller inflow at $r / R=0.7$ of the generic tanker model in selfpropulsion condition: the angle of attack onto the blade (top) and inflow velocity magnitude non-dimensionalized by $U$ (bottom). 
1

2

3

4

5

6

7

8

9
10

11

12

13

14

15

16

17

18

19

20

21

22

23

24

25

26

27

28

29

30

31

32

33

34

35

36

37

38

39

40

41

42

43

44

45

46

47

48

49

50

51

52

53

54

55

56

57

58

59

60

61

62

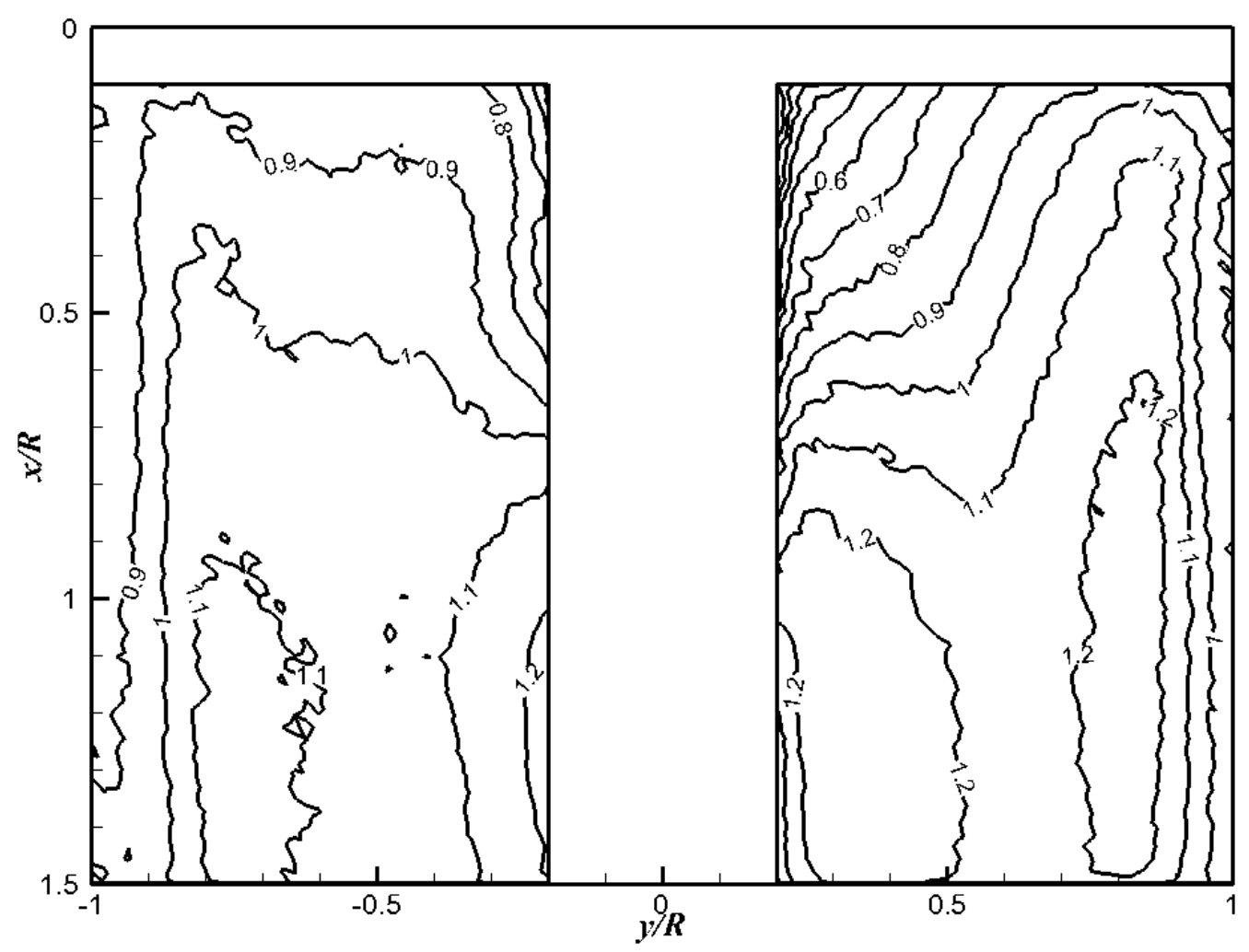

Figure 20. $\overline{\boldsymbol{u}_{\boldsymbol{h}}} / \boldsymbol{U}$ contours on the horizontal plane $(z / R=0)$ of the generic tanker model in self-propulsion condition. 


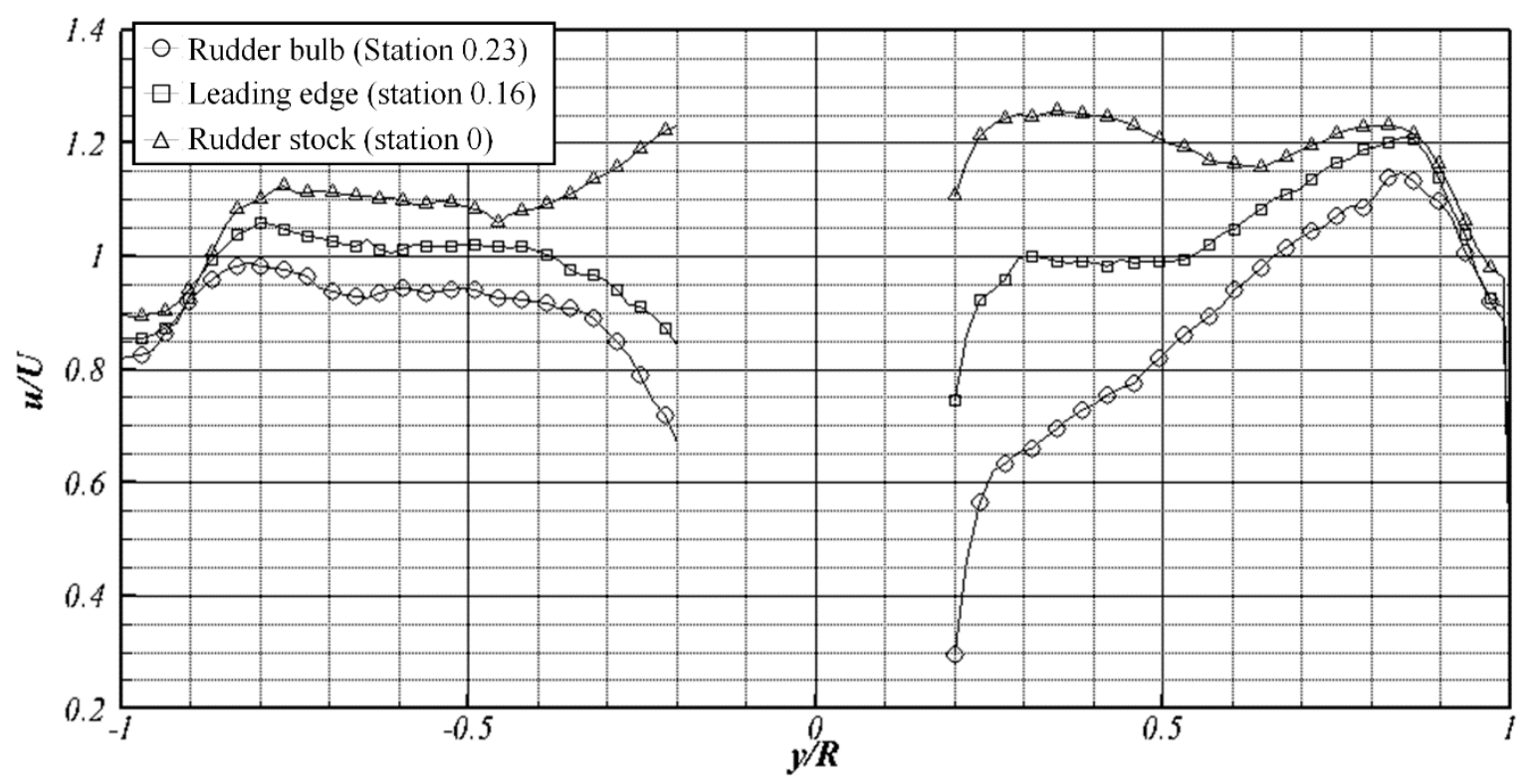

Figure 21. $\overline{\boldsymbol{u}_{\boldsymbol{h}}} / \boldsymbol{U}$ distributions of a horizontal wake field of the generic tanker model in selfpropulsion condition $(z / R=0)$. 


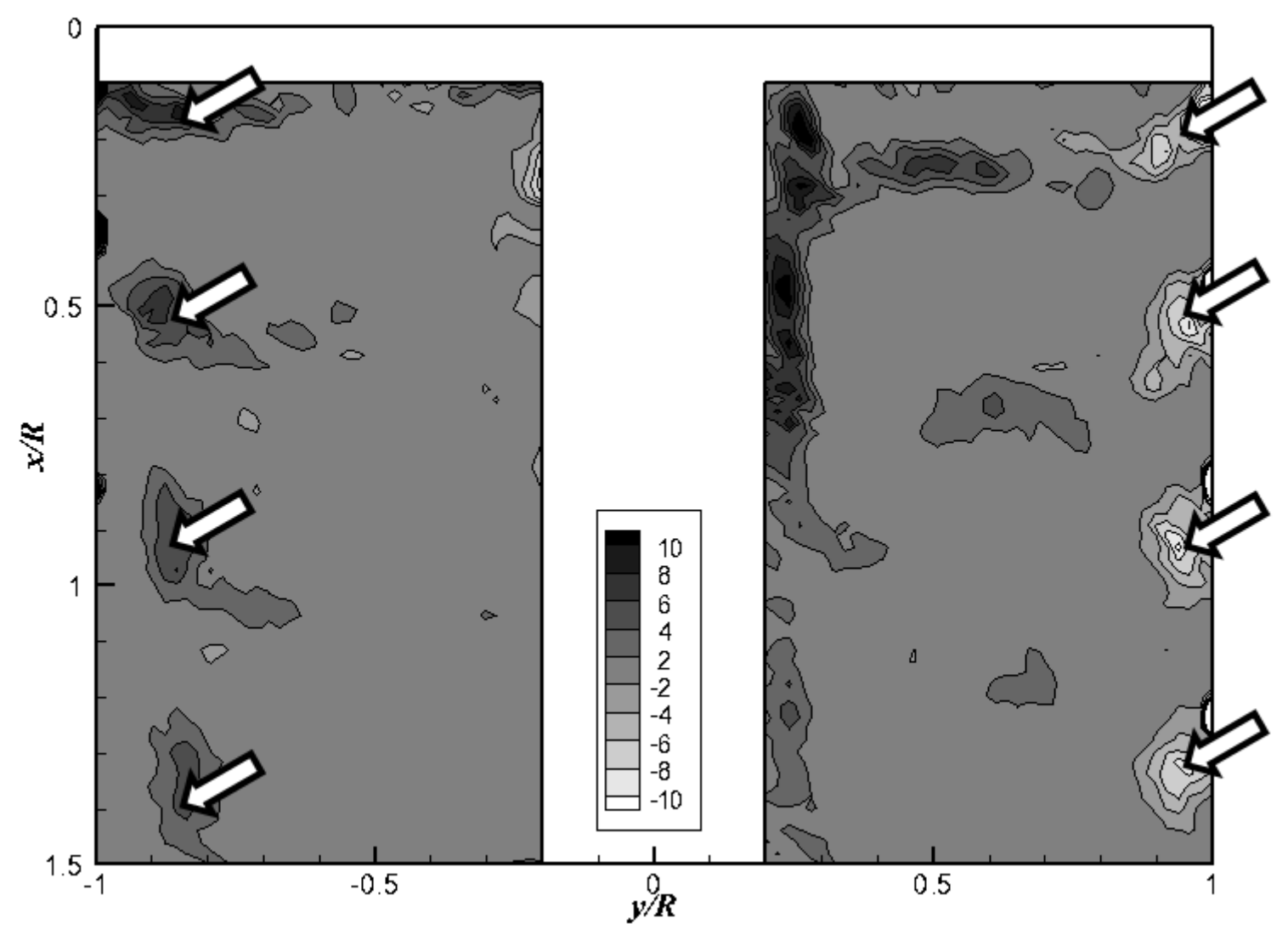

Figure 22. Phase-averaged horizontal wake plane of the generic tanker model in selfpropulsion condition $(z / R=0): \zeta_{x} R / U$ contours and locations of tip vortices (indicated by arrows). 


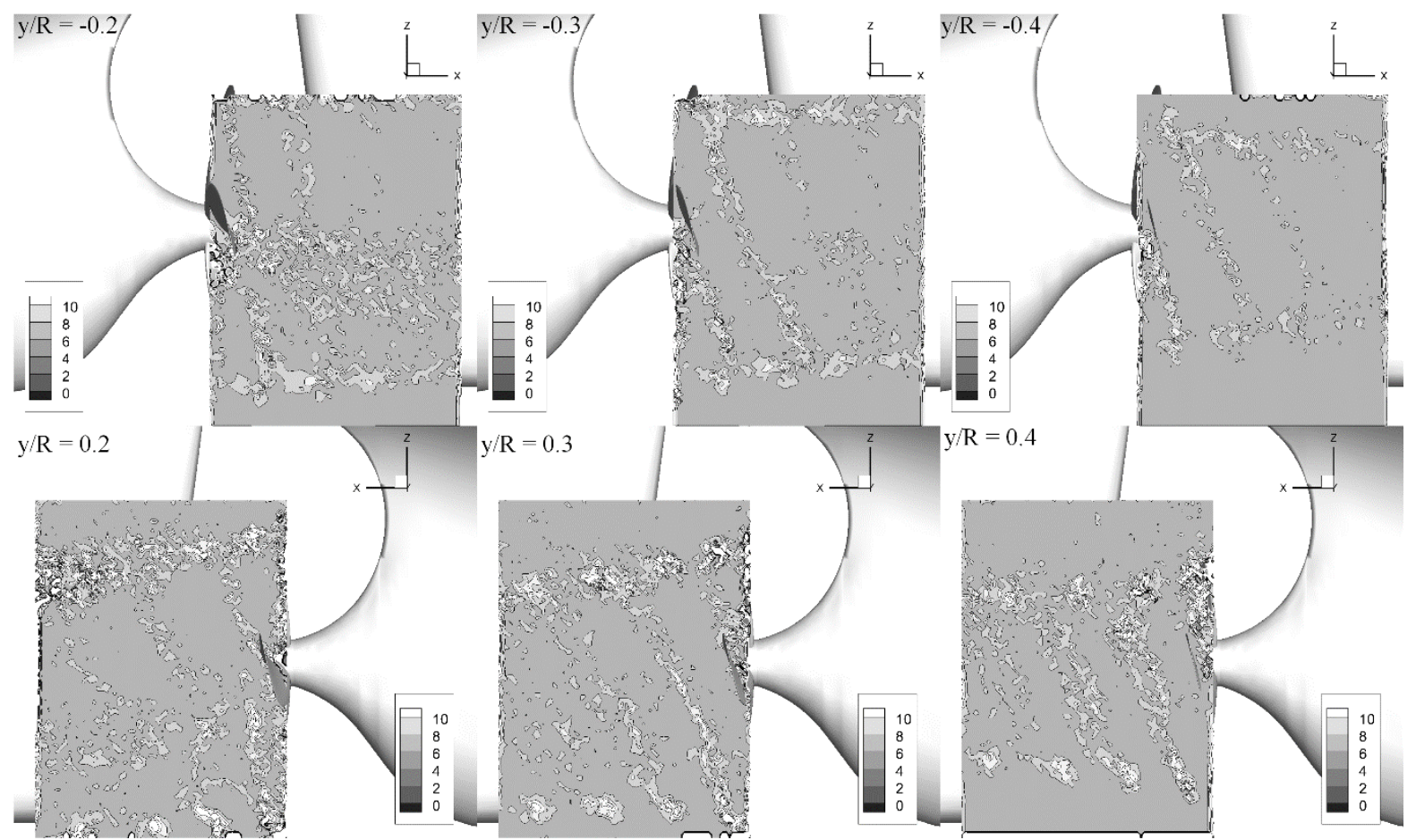

Figure 23. Phase-averaged vertical wake plane of the generic tanker model in self-propulsion condition: magnitude of $\zeta_{y} R / U$ contours. 


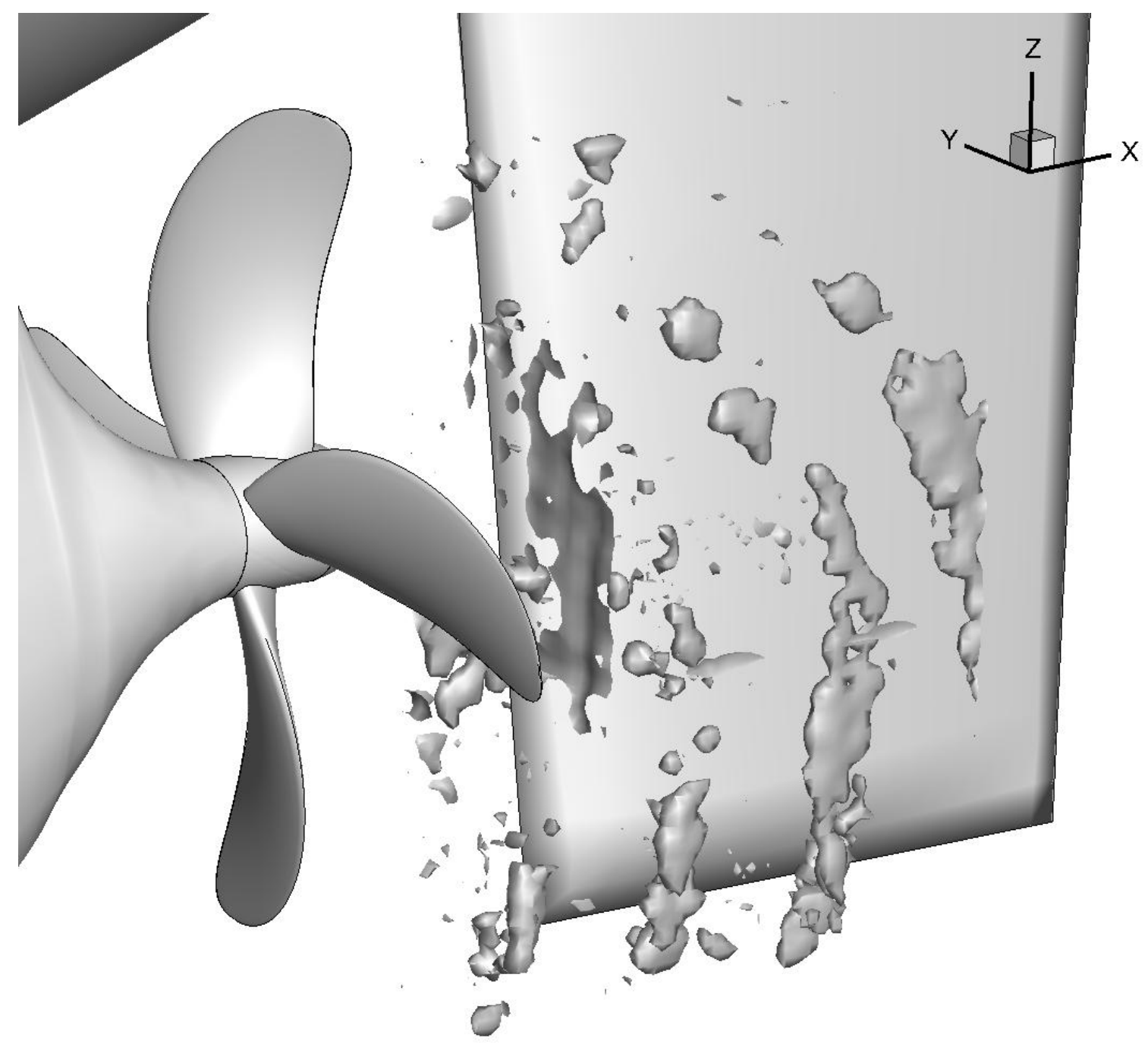

Figure 24. Phase-averaged wake field of the generic tanker model: tip vortex trajectory identified by the iso-surfaces of helicity. 


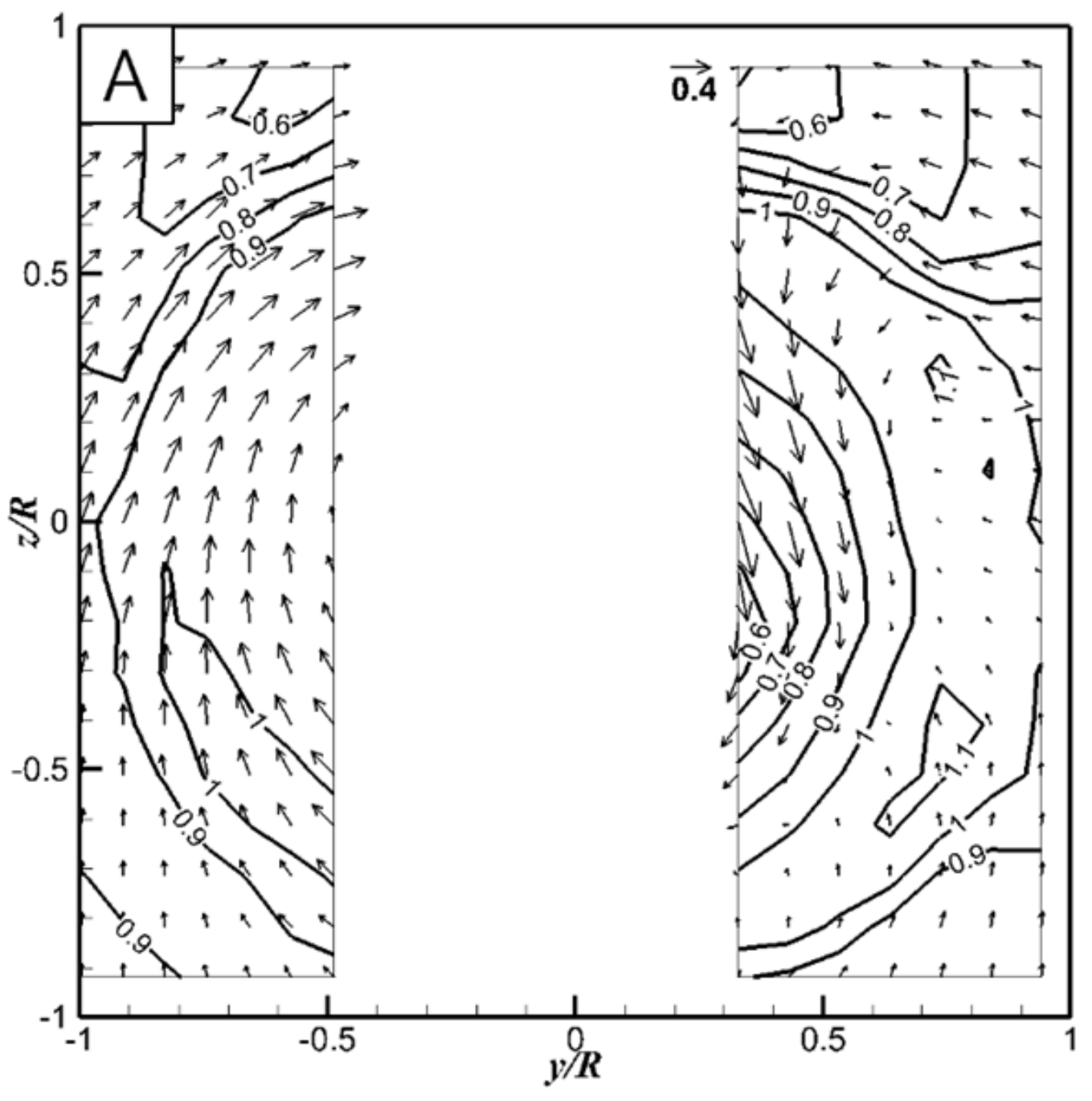




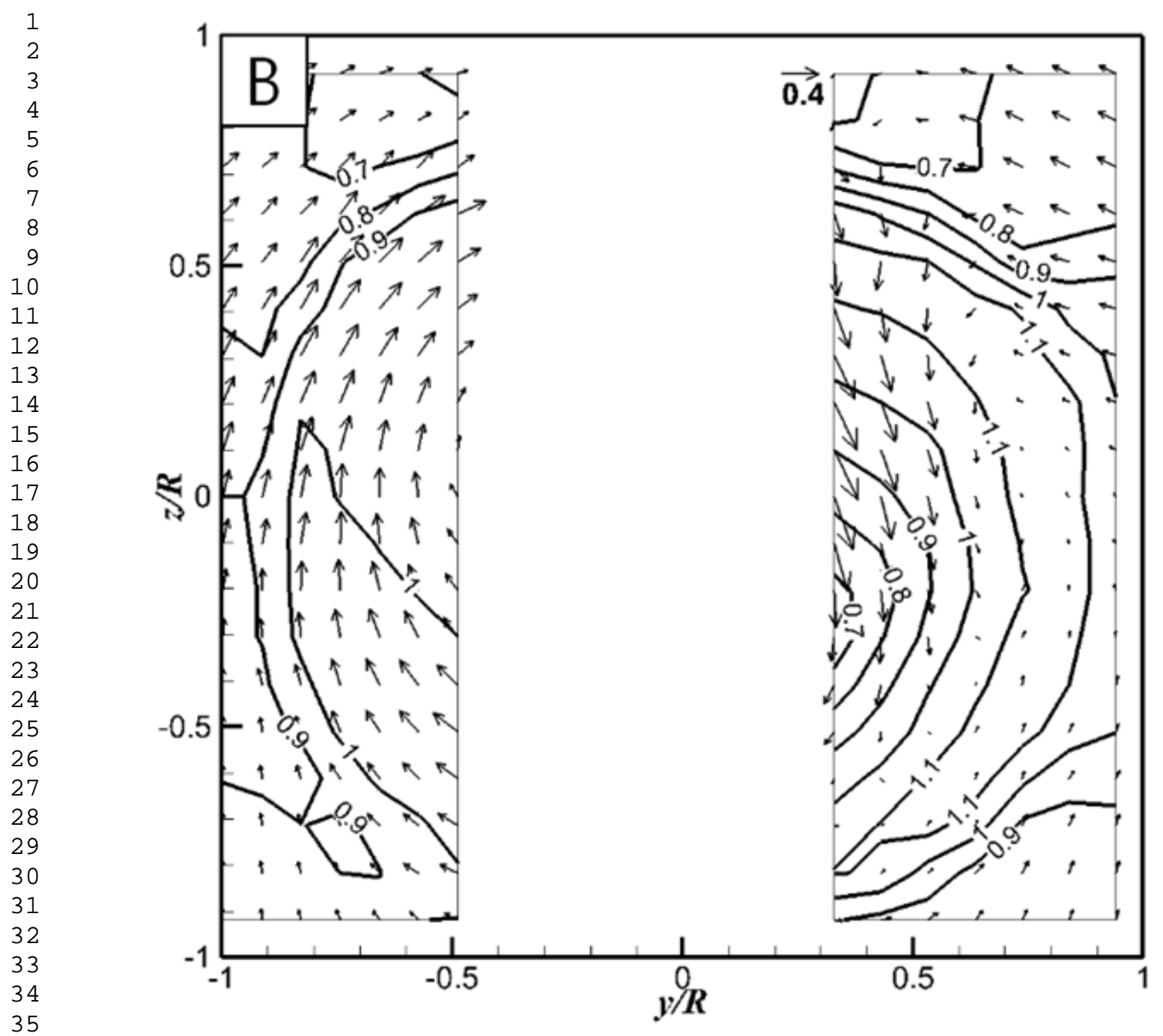

36

37

38

39

40

41

42 
1

2

3

4

5

6

7

8

9
10

11

12

13

14

15

16

17

18

19

20

21

22

23

24

25

26

27

28

29

30

31

32

33

34

35

36

37

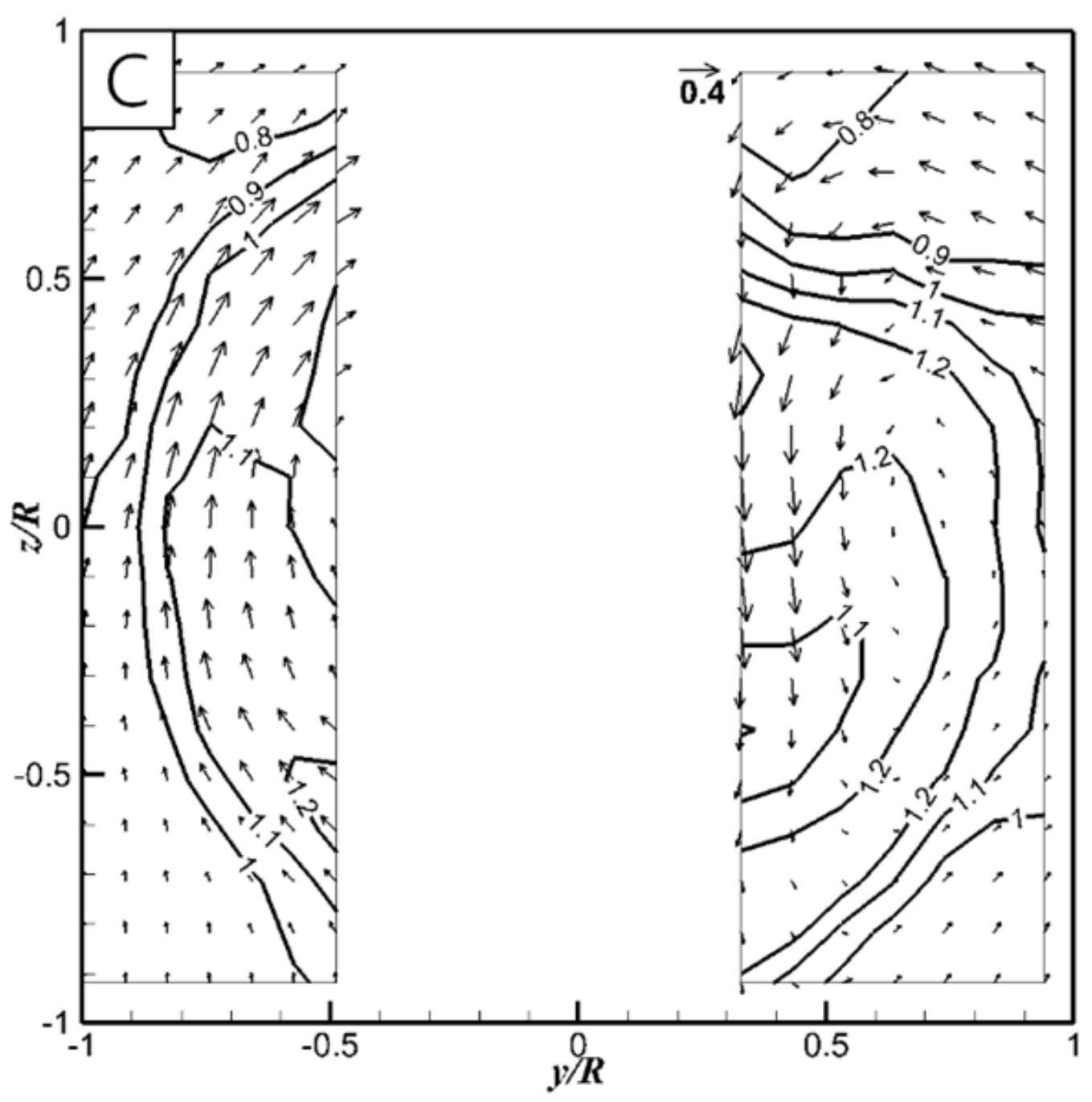

Figure 25. Phase-averaged wake field of the generic tanker model in self-propulsion condition: $\overline{\boldsymbol{u}} / \boldsymbol{U}$ contours and $\overline{\boldsymbol{v}} / \boldsymbol{U}-\overline{\boldsymbol{w}} / \boldsymbol{U}$ vectors (A: station 0.23 , B: station 0.16 , and $\mathrm{C}$ : station 0). 
1

2
3

4

5

6

7

8

9
10

11

12

13

14

15

16

17

18

19

20

21

22

23

24

25

26

27

28

29

30

31

32

33

34

35

36

37

38

39

40

41

42

43

44

45

46

47

48

49

50

51

52

53

54

55

56

57

58

59

60

61

62

63

64

65

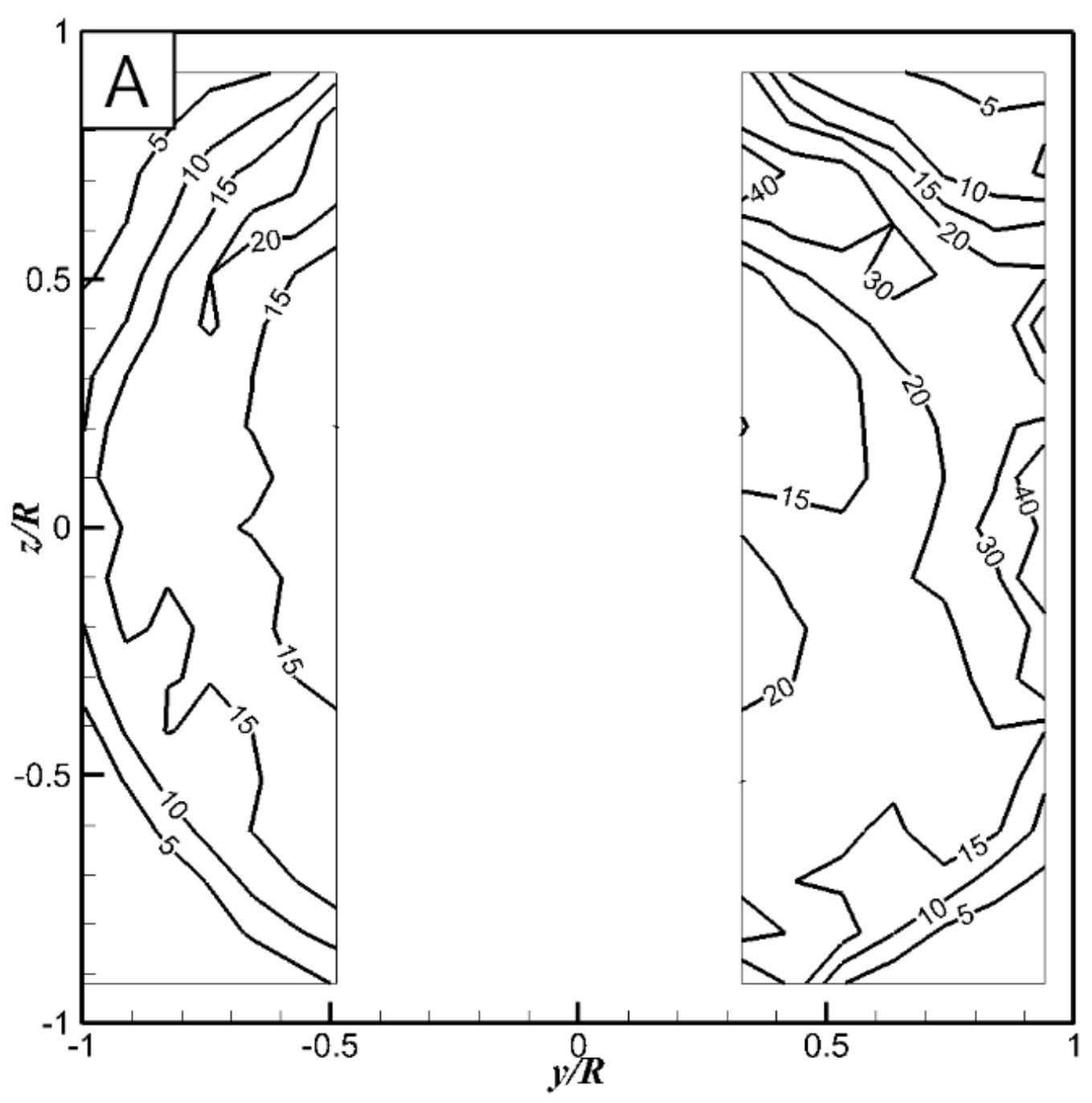


1

2
3

4

5

6

7

8

9
10

11

12

13

14

15

16

17

18

19

20

21

22

23

24

25

26

27

28

29

30

31

32

33

34

35

36

37

38

39

40

41

42

43

44

45

46

47

48

49

50

51

52

53

54

55

56

57

58

59

60

61

62

63

64

65

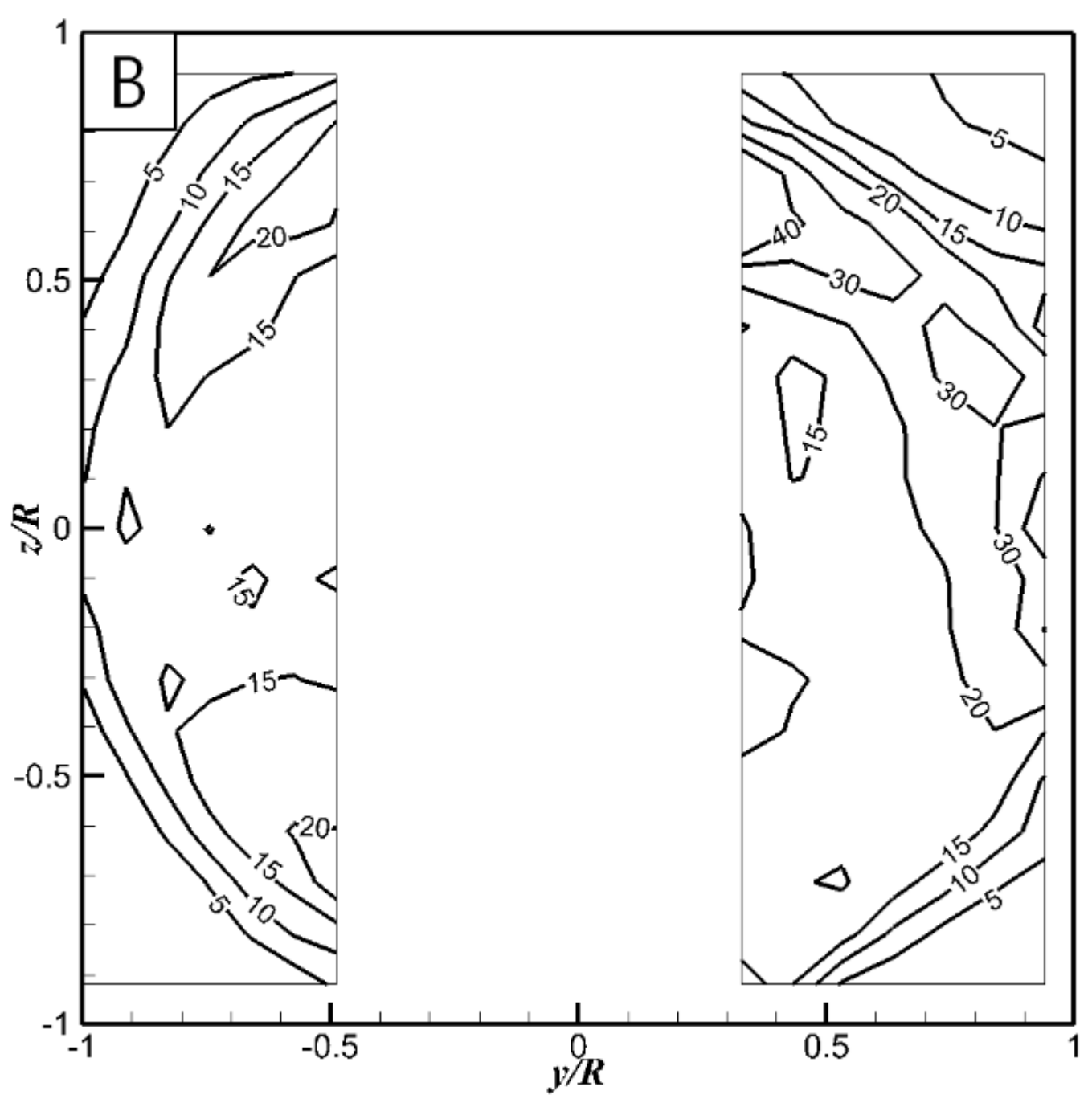


1

2

3

4

5

6

7

8

9

10

11

12

13

14

15

16

17

18

19

20

21

22

23

24

25

26

27

28

29

30

31

32

33

34

35

36

37

38

39

40

41

42

43

44

45

46

47

48

49

50

51

52

53

54

55

56

57

58

59

60

61

62

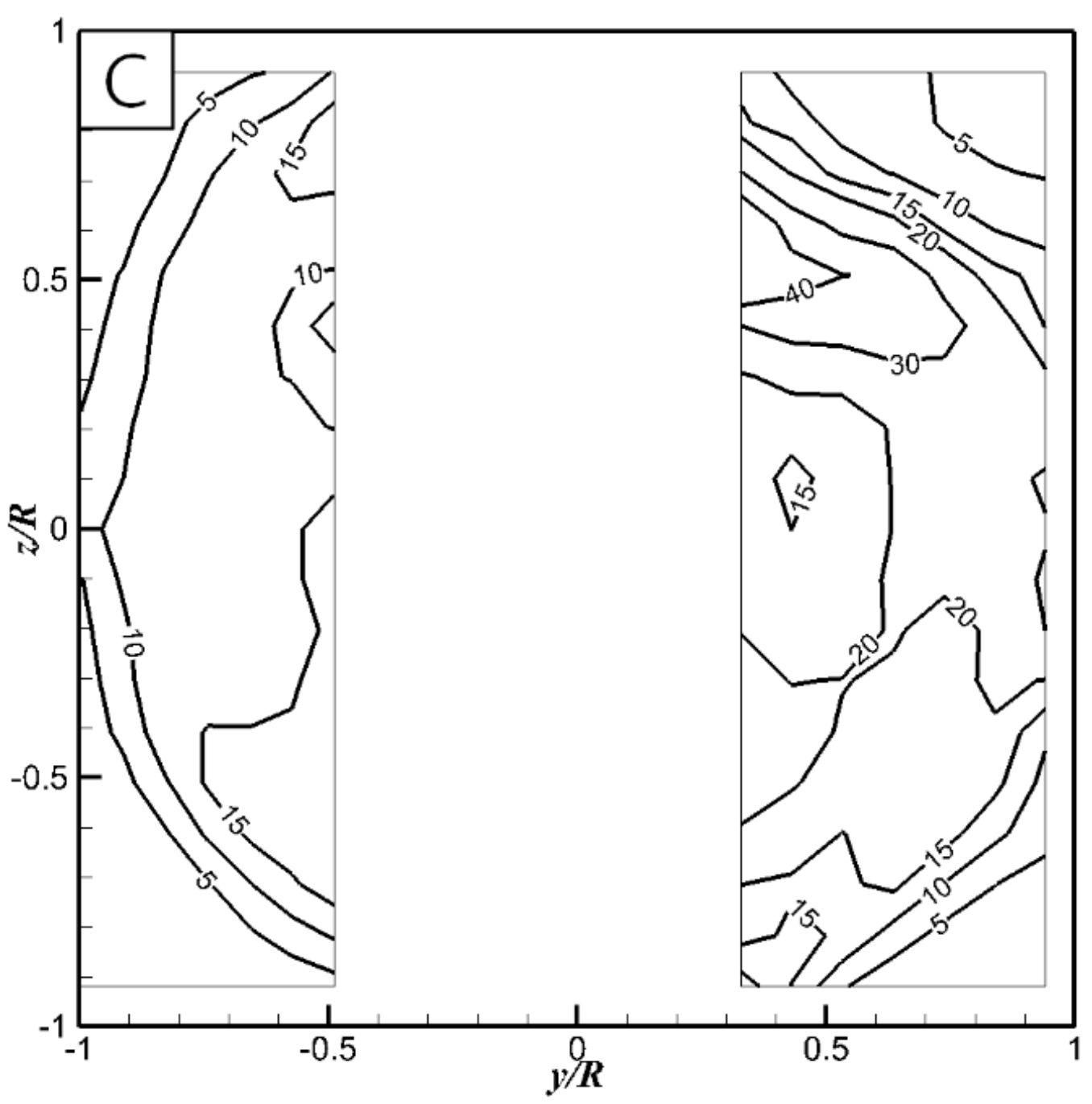

Figure 26. Phase-averaged wake field of the generic tanker model in self-propulsion condition: $\boldsymbol{k} / \boldsymbol{U}^{2} \times \mathbf{1 0 0 0}$ contours (A: station 0.23 , B: station 0.16 , and C: station 0 ). 